\title{
Analysis of DC Electrical Conductivity Models of Carbon Nanotube-Polymer Composites with Potential Application to Nanometric Electronic Devices
}

\author{
Rafael Vargas-Bernal, ${ }^{1}$ Gabriel Herrera-Pérez, ${ }^{1}$ Ma. Elena Calixto-Olalde, ${ }^{1}$ \\ and Margarita Tecpoyotl-Torres ${ }^{2}$ \\ ${ }^{1}$ Departamento de Ingeniería en Materiales, Instituto Tecnológico Superior de Irapuato, Carretera Irapuato Silao Km. 12.5, \\ 36821 Irapuato, Guanajuato, Mexico \\ ${ }^{2}$ Centro de Investigación en Ingeniería y Ciencias Aplicadas (CIICAp), Universidad Autónoma del Estado de Morelos, \\ Avenida Universidad No. 1001, Col. Chamilpa, 62209 Cuernavaca, Morelos, Mexico
}

Correspondence should be addressed to Rafael Vargas-Bernal; rvargasbernal@gmail.com

Received 15 December 2012; Accepted 28 January 2013

Academic Editor: Esteban Tlelo-Cuautle

Copyright (C) 2013 Rafael Vargas-Bernal et al. This is an open access article distributed under the Creative Commons Attribution License, which permits unrestricted use, distribution, and reproduction in any medium, provided the original work is properly cited.

The design of nanometric electronic devices requires novel materials for improving their electrical performance from stages of design until their fabrication. Until now, several DC electrical conductivity models for composite materials have been proposed. However, these models must be valued to identify main design parameters that more efficiently control the electrical properties of the materials to be developed. In this paper, four different models used for modeling DC electrical conductivity of carbon nanotubepolymer composites are studied with the aim of obtaining a complete list of design parameters that allow guarantying to the designer an increase in electrical properties of the composite by means of carbon nanotubes.

\section{Introduction}

In the nanometer era, VLSI circuits must be simulated by novel models that allow us to predict and estimate the electrical behavior waited from their phase of design and during their performance. With the introduction of the nanomaterials as an alternative to silicon, numerous research groups around the world have been developing models to simulate electrical properties of such materials. Within this class of nanomaterials, carbon nanotube-polymer nanocomposites are offering improved electrical properties thanks to inherent electrical properties of the carbon nanotubes either semiconducting or conducting. Previous studies of these materials have predicted successful technological applications [1-3]. However, their design must overcome important barriers involved with the control of phenomena between their interfaces and fabrication methods with the aim of achieving repetitive results.
Electrical properties of materials are determined through four fundamental parameters called dielectric constant, tangent of dielectric loss angle, dielectric breakdown, and electrical conductivity. Most polymers exhibit a highly insulating dielectric behavior and a very high electrical resistivity. Thus, it is required adding an electrically conductive filler to polymers for changing their electrical properties. The resulting nanocomposite of such combination can give place to a semiconducting or conducting behavior. Electrical fillers can be ceramic materials, metals, and/or carbon nanotubes. In particular, carbon nanotubes form composite materials which can be applied in gas sensors, biosensors, electromagnetic shielding, antistatic coatings, high-strength low-density corrosion-resistant components, and lightweight energy storage. The use of these nanocomposites will be extended to VLSI circuit design when a complete domain of electrical properties and fabrication methods can be achieved. In addition, fabrication of these materials must 


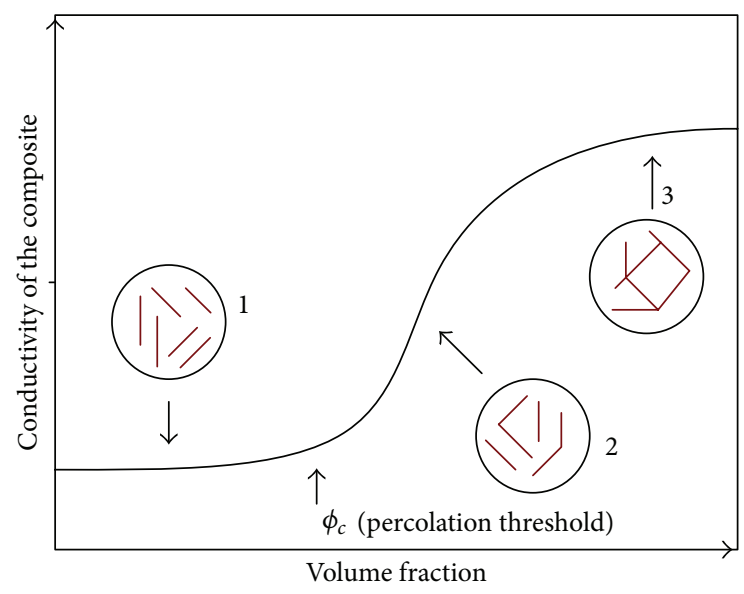

FIGURE 1: Dependence of electrical conductivity on filler volume fraction.

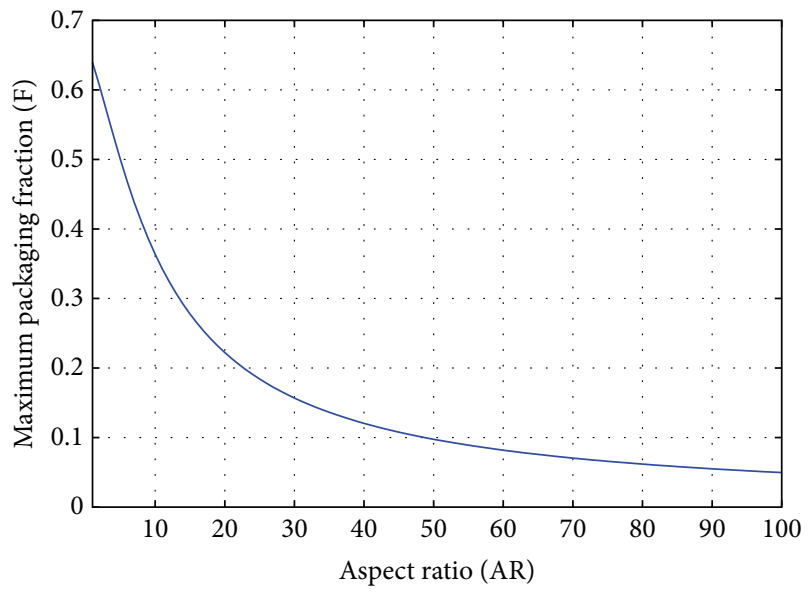

FIGURE 2: Maximum packaging fraction of the filler used in the composite versus aspect ratio of the filler involved.

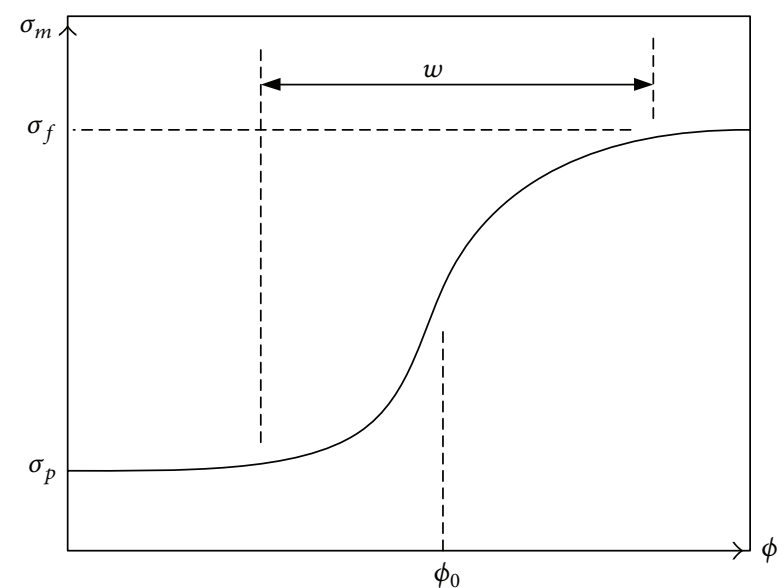

FIGURE 3: Sigmoidal function model for DC electrical conductivity for nanocomposites.

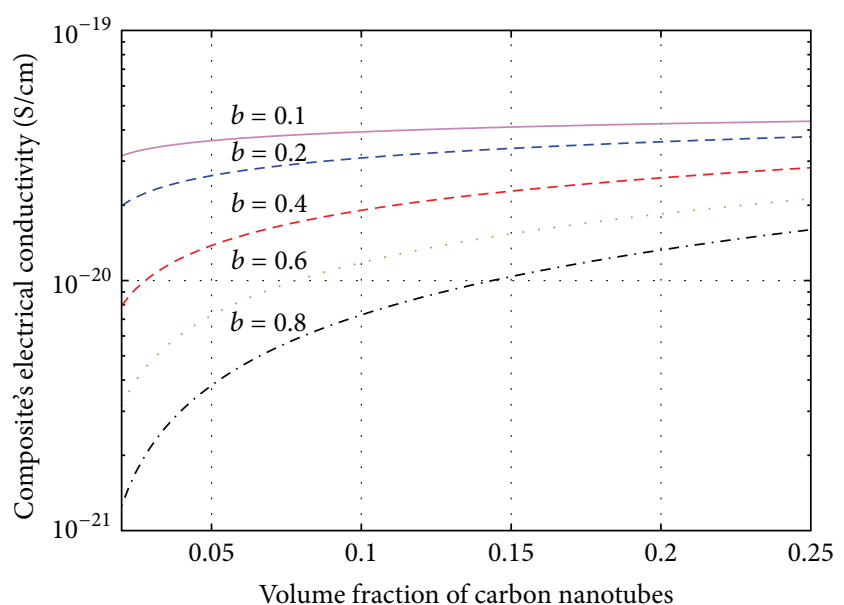

FIGURE 4: DC electrical conductivity of the nanocomposite when the critical exponent of the percolation is varied (Kirkpatrick's model).

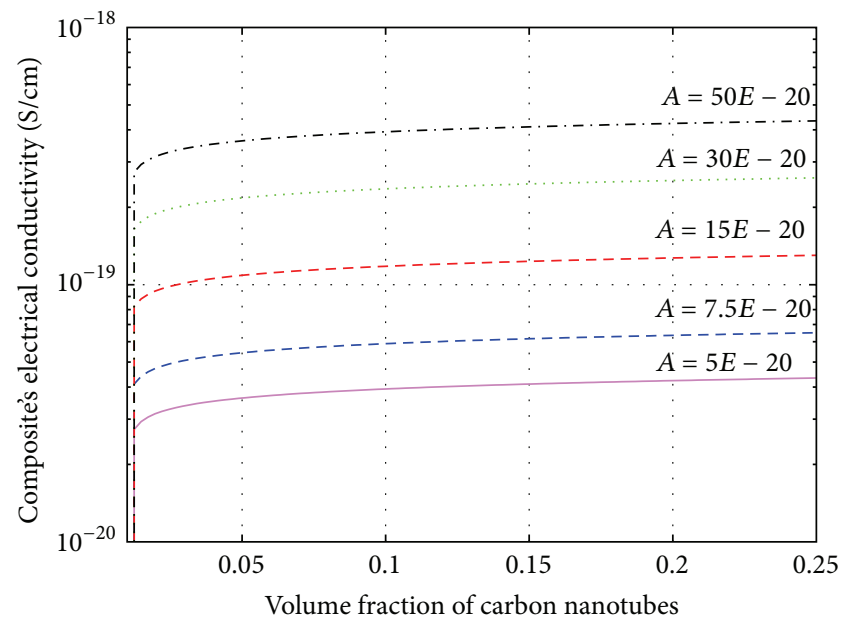

FIGURE 5: DC electrical conductivity of the nanocomposite at varying the conductivity of the filler (Kirkpatrick's model).

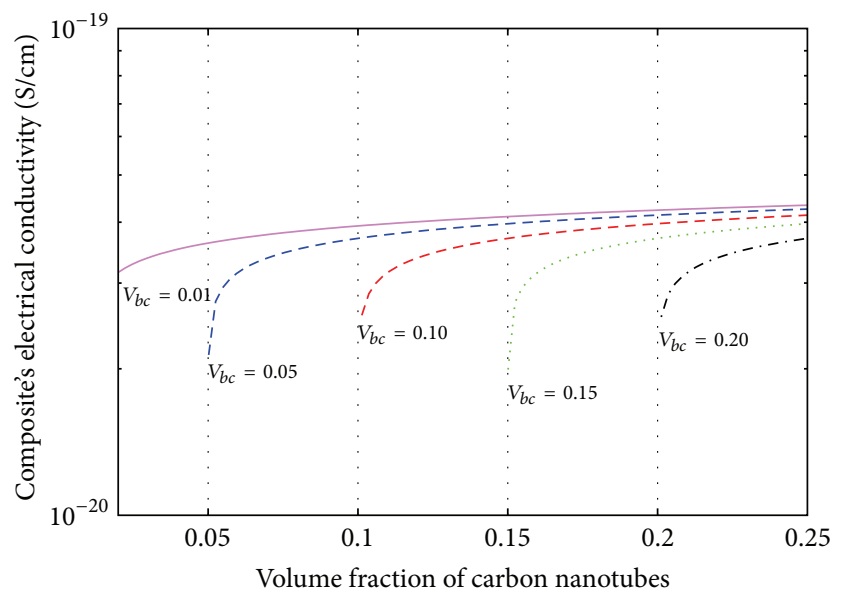

FIGURE 6: DC electrical conductivity of the nanocomposite when the percolation threshold of the filler is varied (Kirkpatrick's model). 


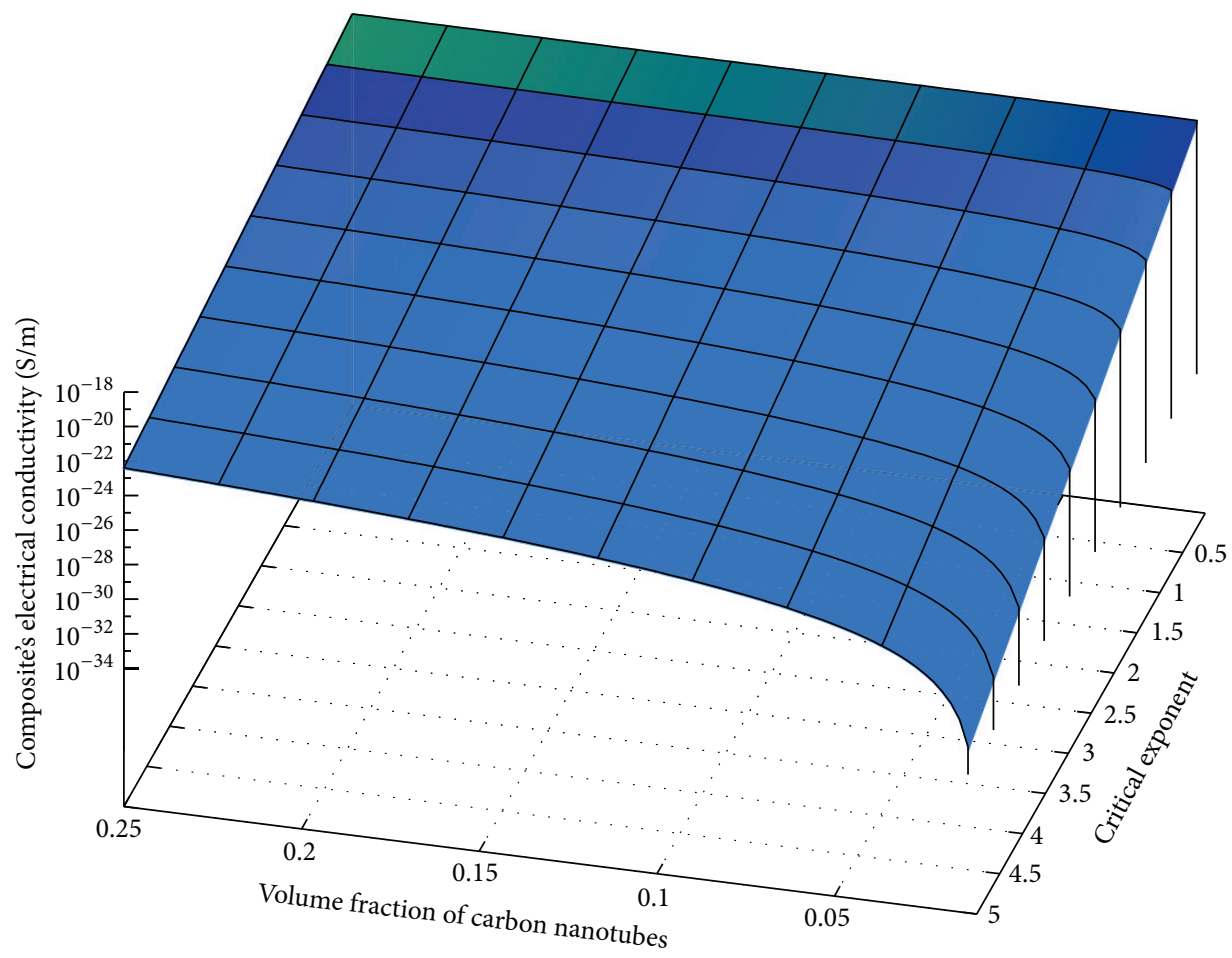

FIGURE 7: DC electrical conductivity of the nanocomposite at varying both the critical exponent of the percolation and the volume fraction of the filler (Kirkpatrick's model).

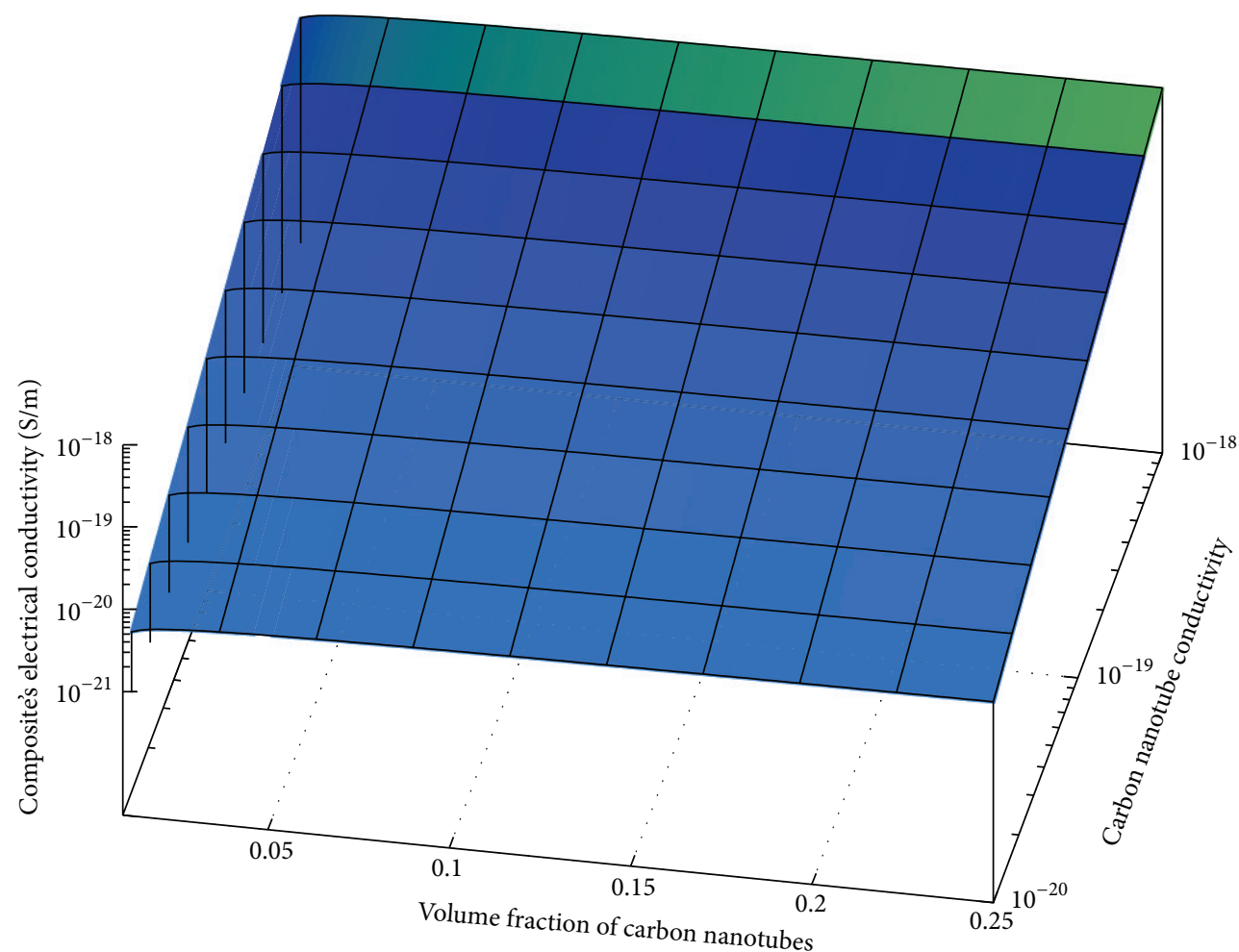

FIGURE 8: DC electrical conductivity of the nanocomposite when both the conductivity of the filler and the volume fraction of the filler are varied (Kirkpatrick's Model). 


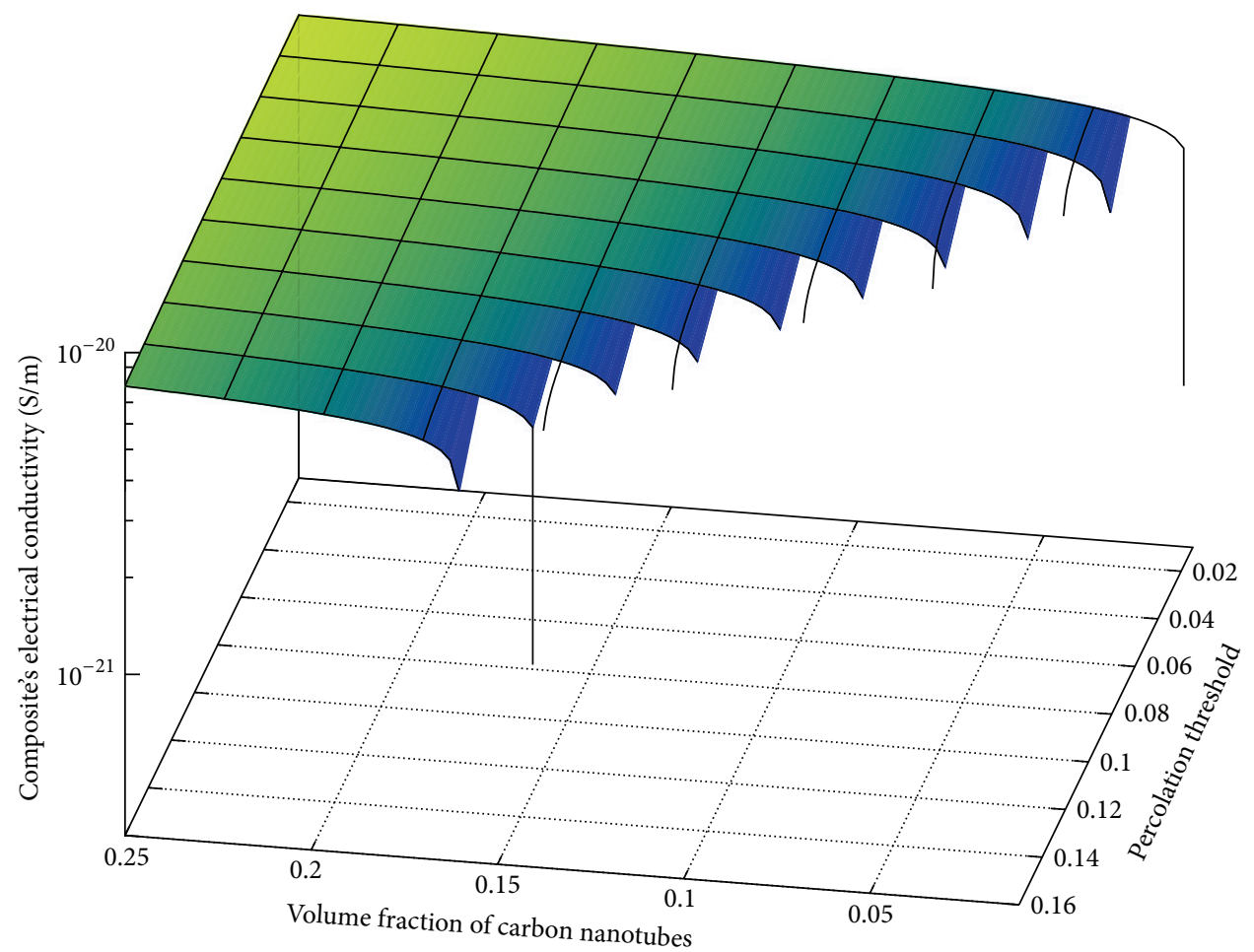

FIGURE 9: DC electrical conductivity of the nanocomposite at varying both the percolation threshold of the filler and the volume fraction of the filler (Kirkpatrick's model).

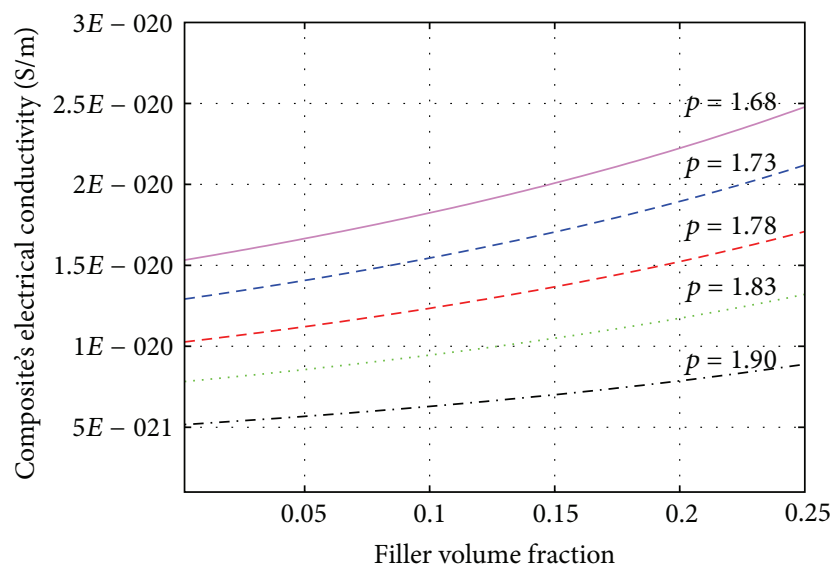

Figure 10: DC electrical conductivity of the nanocomposite when the critical exponent of the percolation is varied (McLachlan's model).

be repetitive to be exploited by electronic industry. Several models of DC electrical behavior have been proposed until now; unfortunately, a complete comparative study among these models that determines a strategic methodology of design does not exist.

In this paper, an explorative study of the DC electrical models of nanocomposites is realized with the aim of obtaining a complete list of design parameters to achieve

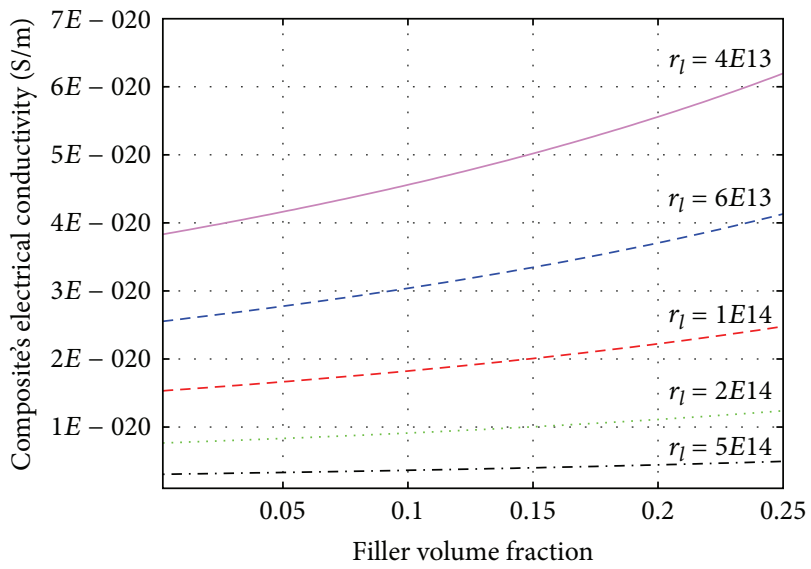

FIGURE 11: DC electrical conductivity of the nanocomposite at varying the conductivity of the matrix (McLachlan's model).

electrical behavior well defined. In Section 2, the description of four DC electrical models for composites, which will be modeled, is realized. Next, these models are simulated with the aim of identifying main design parameters that increase electrical conductivity in Section 3. In Section 4, results of the simulation and their discussion are analyzed with details. Finally, conclusions highlighting the importance of the study realized are given in Section 5. 


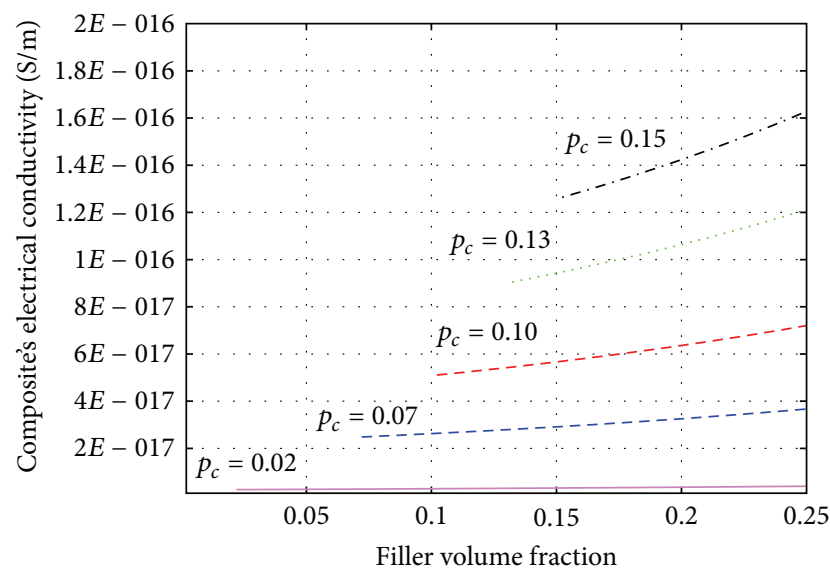

FIGURE 12: DC electrical conductivity of the nanocomposite when the percolation threshold of the filler is varied (McLachlan's model).

\section{DC Electrical Models for Composites}

An electrical model is a mathematical description that represents electrical behavior of an electrical device or system. Direct current (DC) is the unidirectional flow of electric charge. Direct current can flow through metals, semiconductors, insulators, and composites. The contribution to electrical conductivity of the polymer (or matrix) by part of the filler is not continuous and linear, rather it is discrete and nonlinear. There is a critical composition or also called percolation threshold, at which electrical conduction is increased by several orders of magnitude leading the composite from an insulating range to semiconductive and conductive ranges. In this paper, DC electrical models that will be analyzed are: Kirkpatrick's model, McLachlan's model, Mamunya's model, and Sigmoidal function model. The models are associated with an extended basic statistical percolation theory, and the last model makes use of a nonlinear regression.

2.1. Kirkpatrick's Model. This model predicts the DC electrical conductivity based on the likelihood of contact between particles of filler within the composite [3-6]. Its equation is expressed as a power law equation of the form

$$
\sigma_{m}=A\left(\phi-V_{b c}\right)^{b}
$$

where $\sigma_{m}$ is the conductivity of the composite, $A$ is the conductivity of the filler, $\phi$ is the volume fraction of filler, $V_{b c}$ is the percolation threshold of filler, and $b$ is the critical exponent which depends on the type of space dimension, and it is a characteristic value experimentally obtained. The percolation threshold is the minimum quantity of the filler required to form a continuous network of particles (as shown in Figure 1) to transport electrical charge between ends of the material being electrically polarized by a DC source. As it is illustrated in this figure, there are three main zones in which conductivity of nanocomposites can be found. At low filler concentrations, zone 1 in Figure 1, the conductivity of the composite is very close to the pure polymer matrix. In zone 2 , the percolation threshold have been achieved and the electrical conductivity undergoes a drastic increase due to the start of the formation and consolidation of an electrically conductive network through the sample. Finally, in zone 3 , the maximum electrical conductivity is obtained when a completely interconnected network is formed.

2.2. McLachlan's Model. Some physical models can describe the macroscopic properties of a medium in accordance with their properties and the relative fractions of its components under the name of effective medium approximations or effective medium theory. The properties under study, in particular, are usually the electrical conductivity or the dielectric constant of the medium. McLachlan et al. [7-11] proposed a statistical model of the DC electrical conductivity of the composite materials based on the effective medium theory by means of the equation

$$
\frac{(1-\phi)\left(\rho_{m}^{1 / p}-\rho_{f}^{1 / p}\right)}{\rho_{m}^{1 / p}+\left(\left(1-p_{c}\right) / p_{c}\right) \rho_{f}^{1 / p}}+\frac{\phi\left(\rho_{m}^{1 / p}-r_{l}^{1 / p}\right)}{\rho_{m}^{1 / p}+\left(\left(1-p_{c}\right) / p_{c}\right) r_{l}^{1 / p}}=0,
$$

where $\phi$ is the volume fraction of filler, $p_{c}$ is the percolation threshold of filler, $\rho_{f}$ is the resistivity of the filler, $r_{l}$ is the resistivity of the polymer or matrix, and $p$ is the critical exponent which represents the volume fraction of the phases.

2.3. Mamunya's Model. The model suggested by Mamunya et al. [12-16] makes uses of the surface energy of the polymer and the filler, and it includes the aspect ratio of the filler. In addition, this theory relates the electrical conductivity of composites with the presence of clusters of connected particles. Therefore, there exists a relationship between the electrical conductivity of the composite and the volume fraction of the filler. Their equations are

$$
\log \left(\sigma_{m}\right)=\log (\mathrm{SC})+\left(\log (\mathrm{SF})-\log \left(\sigma_{c}\right)\right)\left(\frac{\phi-\phi_{c}}{\mathrm{~F}-\phi_{c}}\right)^{k},
$$

where

$$
\begin{gathered}
k=\frac{K \phi_{c}}{\left(\phi-\phi_{c}\right)^{3 / 4}}, \\
K=A-B \gamma_{p f}, \\
\gamma_{p f}=\gamma_{p}+\gamma_{f}-2\left(\gamma_{p} \gamma_{f}\right)^{1 / 2}, \\
\mathrm{~F}=\frac{5}{75 /(10+\mathrm{AR})+\mathrm{AR}} .
\end{gathered}
$$

In this model, $\sigma_{m}$ is the conductivity of the composite, SC is the conductivity at the percolation threshold, SF is the conductivity at the maximum packaging fraction $(\mathrm{F}), \phi$ is the volume fraction, $\phi_{c}$ is the percolation threshold, $\gamma_{p f}$ is the interfacial tension between polymer and filler, $\gamma_{p}$ is the surface energy of the polymer, $\gamma_{f}$ is the surface energy of the filler, $A$ and $B$ are constants, and $\mathrm{AR}$ is the aspect ratio. In Figure 2, the maximum packaging fraction $(\mathrm{F})$ has been calculated to illustrate the effect of the aspect ratio of the filler. 


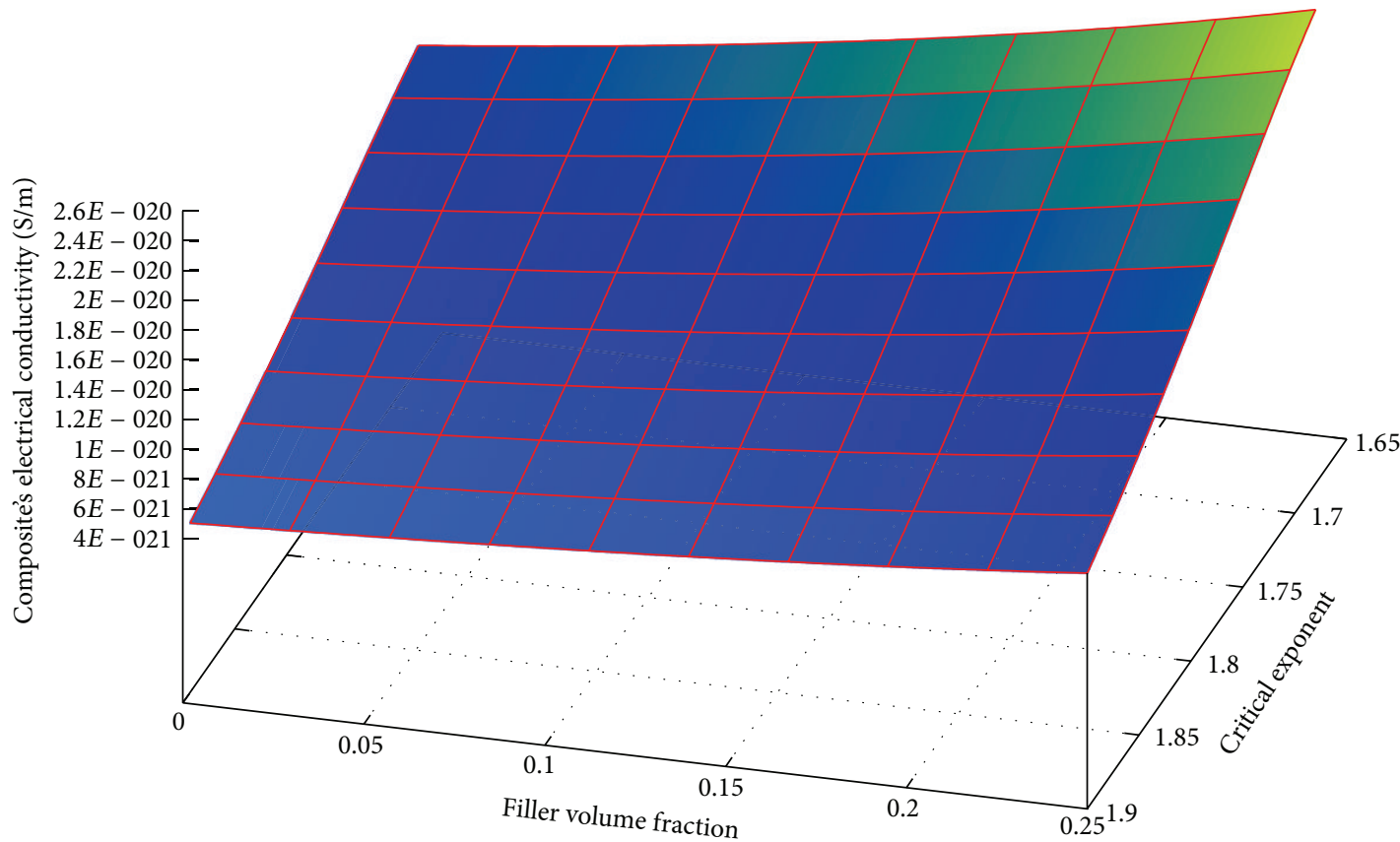

FIGURE 13: DC electrical conductivity of the nanocomposite at varying both the critical exponent of the percolation and the volume fraction of the filler (McLachlan's model).

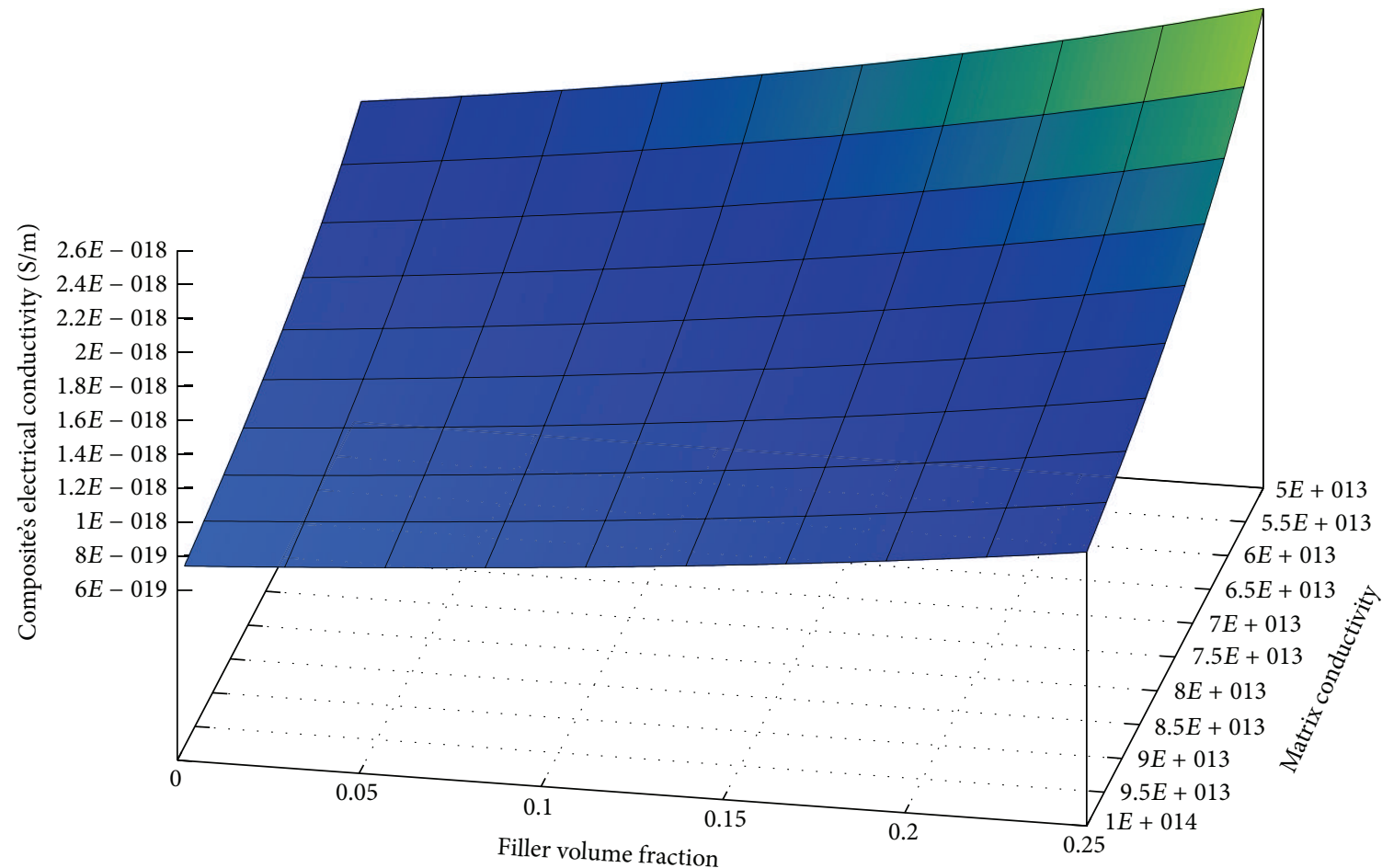

FIGURE 14: DC electrical conductivity of the nanocomposite when both conductivity of the matrix and the volume fraction of the filler are varied (McLachlan's model).

2.4. Sigmoidal Function Model. A sigmoidal function [1721] is a mathematical logistic function with " $S$ " shape and generically defined as

$$
S(t)=\frac{a}{1+e^{(-b t+c)}} .
$$

In particular, the model used in the simulations of DC electrical conductivity of nanocomposites was

$$
\sigma_{m}=\sigma_{p}+\frac{\mathrm{SF}-\sigma_{p}}{1+\exp [-(\phi-\mathrm{PCP}) / w]},
$$




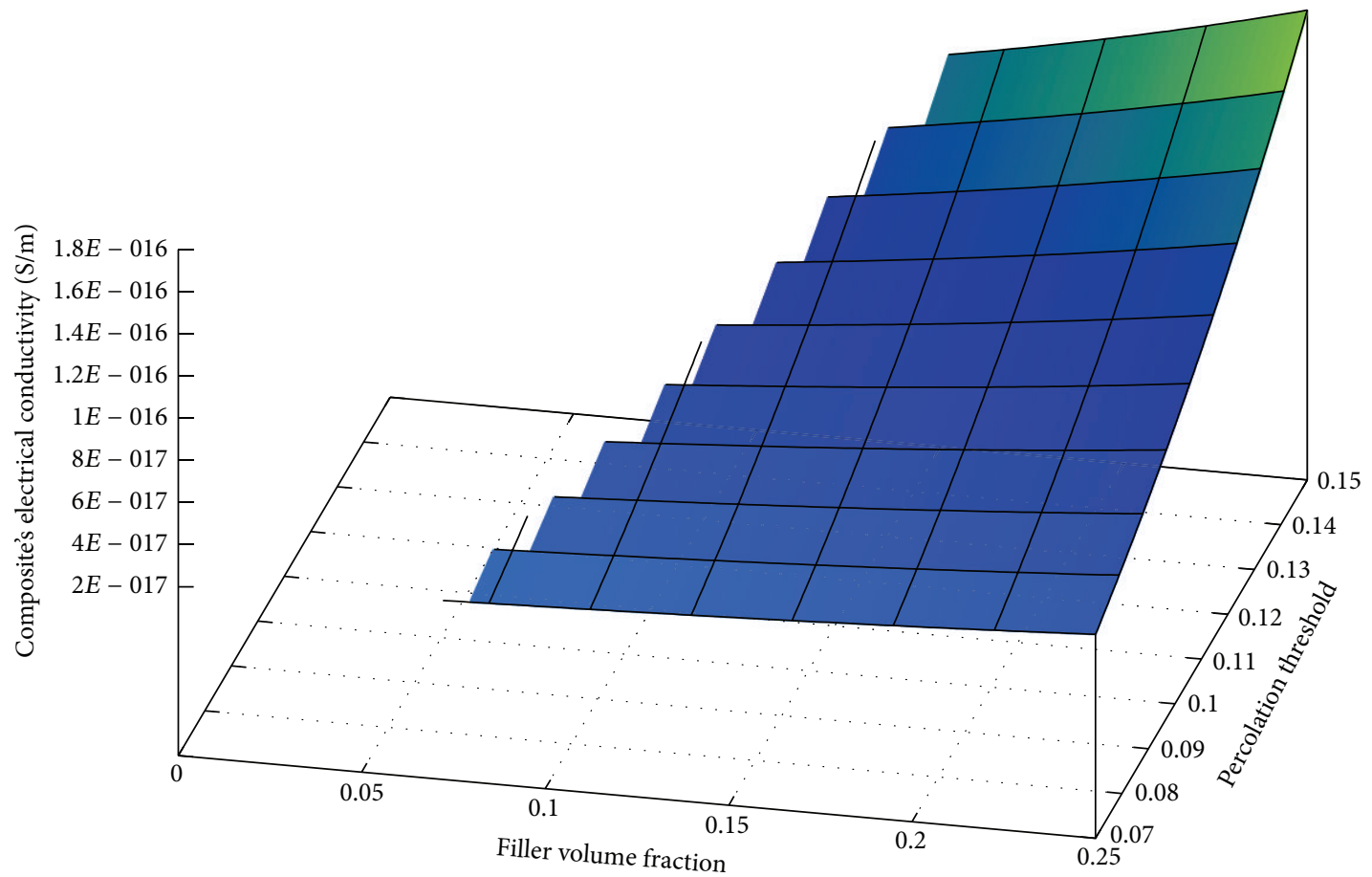

FIGURE 15: DC electrical conductivity of the nanocomposite at varying both the percolation threshold of the filler and the volume fraction of the filler (McLachlan's model).

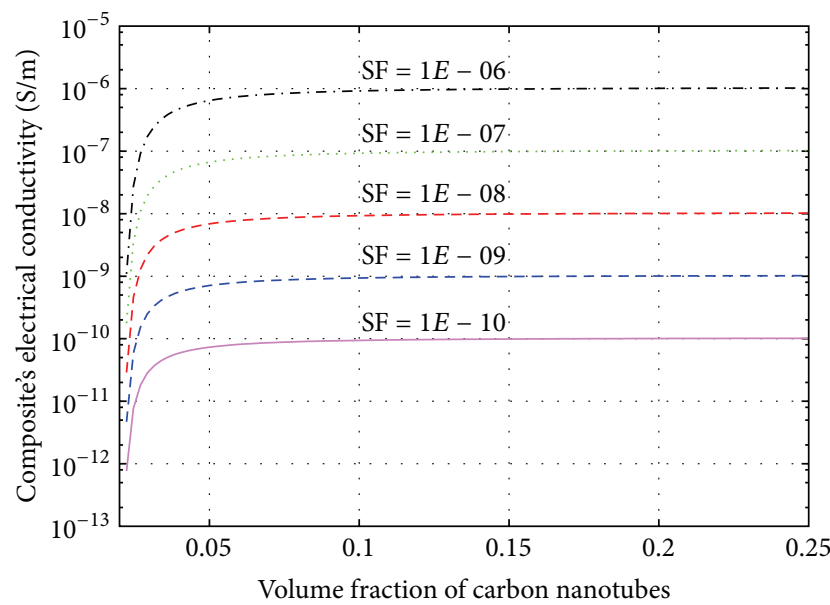

FIGURE 16: DC electrical conductivity of the nanocomposite when the conductivity at the maximum packaging fraction is varied (Mamunya's model).

where $\sigma_{m}$ is the conductivity of the composite, SF is the conductivity of the filler, $\sigma_{p}$ is the conductivity of the polymer, $\phi$ is the volume fraction of the filler, PCP is the volume fraction of the filler in the midpoint of the percolation, and $w$ is the width of the percolation region. Therefore, $\sigma$ represents the independent variable, and the constants can be identified as $a=\mathrm{SF}-\sigma_{p}, b=-1 / w$, and $c=\mathrm{PCP} / w$. This equation is represented in Figure 3.

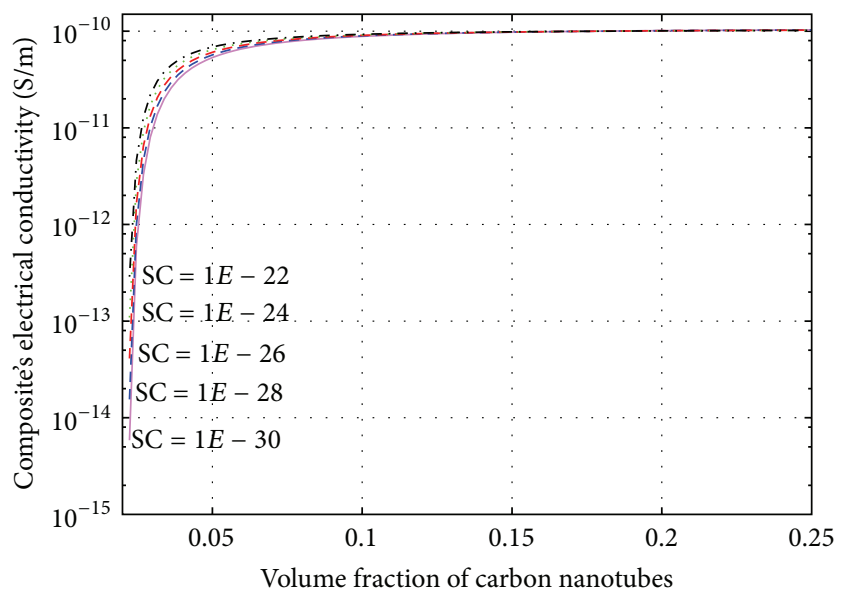

FIgURE 17: DC electrical conductivity of the nanocomposite at varying the conductivity at the percolation threshold (Mamunya's model).

\section{Simulation of the DC Conductivity Models for Nanocomposites}

The first step taken in the analysis of DC electrical conductivity models was evaluating their behavior by means of the variation of their parameters, with the aim of determining which parameters modify in a great proportion the electrical conductivity of the nanocomposites. The models selected for this study include the models reported by Kirkpatrick et al. [3-6], McLachlan et al. [7-11], Mamunya et al. [12-16], and 
TABle 1

\begin{tabular}{|c|c|c|c|c|}
\hline Mathematical models & & Parameters & & $\begin{array}{c}\text { Electrical } \\
\text { conductivity of the } \\
\text { composite materials }\end{array}$ \\
\hline \multirow{4}{*}{ Kirkpatrick's model } & $\begin{array}{c}\text { Conductivity of the } \\
\text { Filler }\end{array}$ & Critical exponent & Percolation threshold & \\
\hline & $5 E-20-50 E-20$ & \multirow{3}{*}{$0.1-0.8$} & & $1 E-20-4.3 E-19$ \\
\hline & & & & $1.4 E-21-4.3 E-19$ \\
\hline & & & $0.01-0.20$ & $2 E-20-4.3 E-19$ \\
\hline \multirow{4}{*}{ McLachlan's model } & $\begin{array}{l}\text { Resistivity of the } \\
\text { matrix }\end{array}$ & Critical exponent & Percolation threshold & \\
\hline & $4 E 13-5 E 14$ & \multirow{3}{*}{$1.68-1.90$} & & $0.3 E-20-6.2 E-20$ \\
\hline & & & & $5 E-21-2.5 E-20$ \\
\hline & & & $0.02-0.15$ & $0.2 E-17-1.63 E-16$ \\
\hline \multirow{4}{*}{ Mamunya's model } & $\begin{array}{l}\text { Conductivity at the } \\
\text { percolation } \\
\text { threshold }\end{array}$ & $\begin{array}{l}\text { Conductivity at the } \\
\text { maximum } \\
\text { packaging fraction }\end{array}$ & \multirow[t]{3}{*}{ Packaging fraction } & \\
\hline & $1 E-30-1 E-22$ & & & $6 E-15-1 E-10$ \\
\hline & & $1 E-10-1 E-06$ & & $9 E-13-1 E-06$ \\
\hline & & & $0.055-0.64$ & $2 E-13-1 E-10$ \\
\hline \multirow{4}{*}{$\begin{array}{l}\text { Sigmoidal function } \\
\text { model }\end{array}$} & $\begin{array}{l}\text { Midpoint of the } \\
\text { percolation region's } \\
\text { width }\end{array}$ & $\begin{array}{l}\text { Conductivity of the } \\
\text { filler }\end{array}$ & $\begin{array}{l}\text { Width of the percolation } \\
\text { region }\end{array}$ & \\
\hline & $0.05-0.20$ & \multirow{3}{*}{$1.5 E-15-1 E-07$} & & $2.8 E-12-1 E-10$ \\
\hline & & & & $8 E-15-1 E-19$ \\
\hline & & & $5 E-03-45 E-03$ & $1.7 E-09-1 E-10$ \\
\hline
\end{tabular}

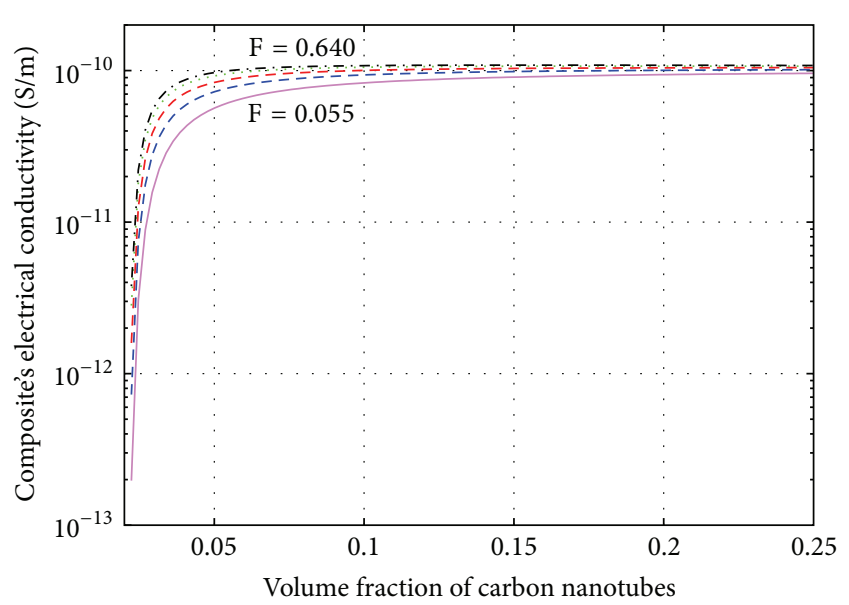

FIGURE 18: DC electrical conductivity of the nanocomposite when the packaging fraction of the filler is varied (Mamunya's model).

Sigmoidal function [17-21]. The functions were evaluated using Gnuplot 4.6.

3.1. Simulations of Kirkpatrick's Model. The evaluation of the Kirkpatrick's model was realized for three parameters: critical exponent, conductivity of the filler, and percolation threshold. The volume fraction was restricted to the range of 0.02 to 0.25 . The critical exponent of the percolation was varied from 0.1 to 0.8 . The conductivity of the filler was varied from $5 E-20$ to $50 E-20 \mathrm{~S} / \mathrm{cm}$. The percolation threshold was varied from 0.01 to 0.20 .

3.2. Simulations of McLachlan's Model. The evaluation of the McLachlan's model was realized for three parameters: critical exponent, conductivity of the matrix, and percolation threshold. The volume fraction was restricted to the range of 0.02 to 0.25 . The critical exponent was varied from 1.68 to $1.90 \mathrm{~S} / \mathrm{cm}$. The resistivity of the matrix was varied from $4 E 13$ to $5 E 14 \mathrm{~S} / \mathrm{cm}$. The percolation threshold was varied from 0.02 to 0.15 .

3.3. Simulations of Mamunya's Model. The evaluation of the Mamunya's model was realized for three parameters: conductivity at the maximum packaging fraction, conductivity at the percolation threshold, and packaging fraction. The volume fraction was restricted to the range of 0.02 to 0.25 . The conductivity at the maximum packaging fraction was varied from $1 E-10$ to $1 E-06 \mathrm{~S} / \mathrm{cm}$. The resistivity at the percolation threshold was varied from $1 E-30$ to $1 E-22 \mathrm{~S} / \mathrm{cm}$. The packaging fraction was varied from 0.055 to 0.64 .

3.4. Simulations of Sigmoidal Function Model. The evaluation of the Sigmoidal function model was realized for three 


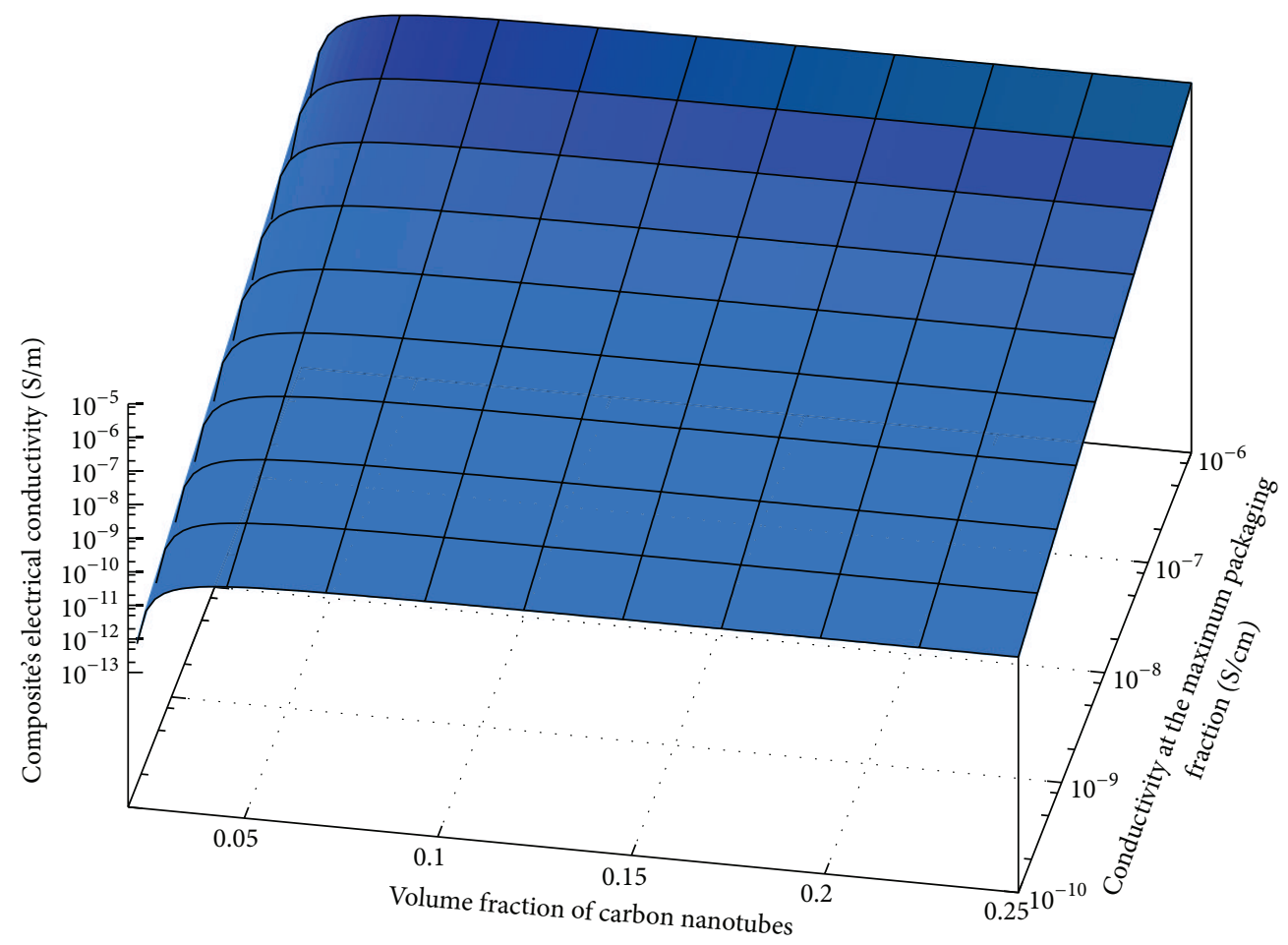

FIGURE 19: DC electrical conductivity of the nanocomposite at varying both the conductivity at the maximum packaging fraction of the filler and the volume fraction of the filler (Mamunyas model).

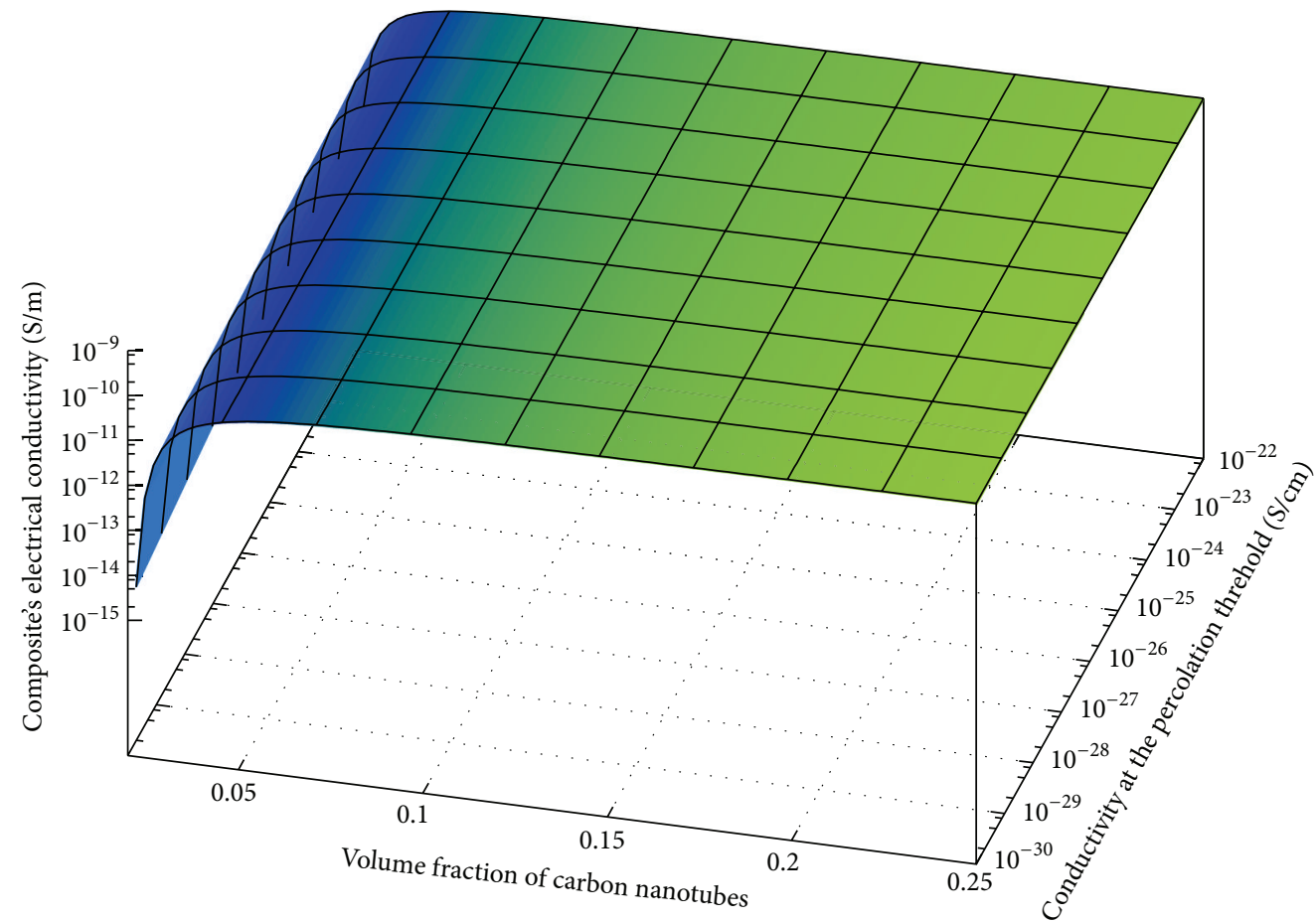

FIGURE 20: DC electrical conductivity of the nanocomposite when both the conductivity at the percolation threshold of the filler and the volume fraction of the filler are varied (Mamunya's model). 


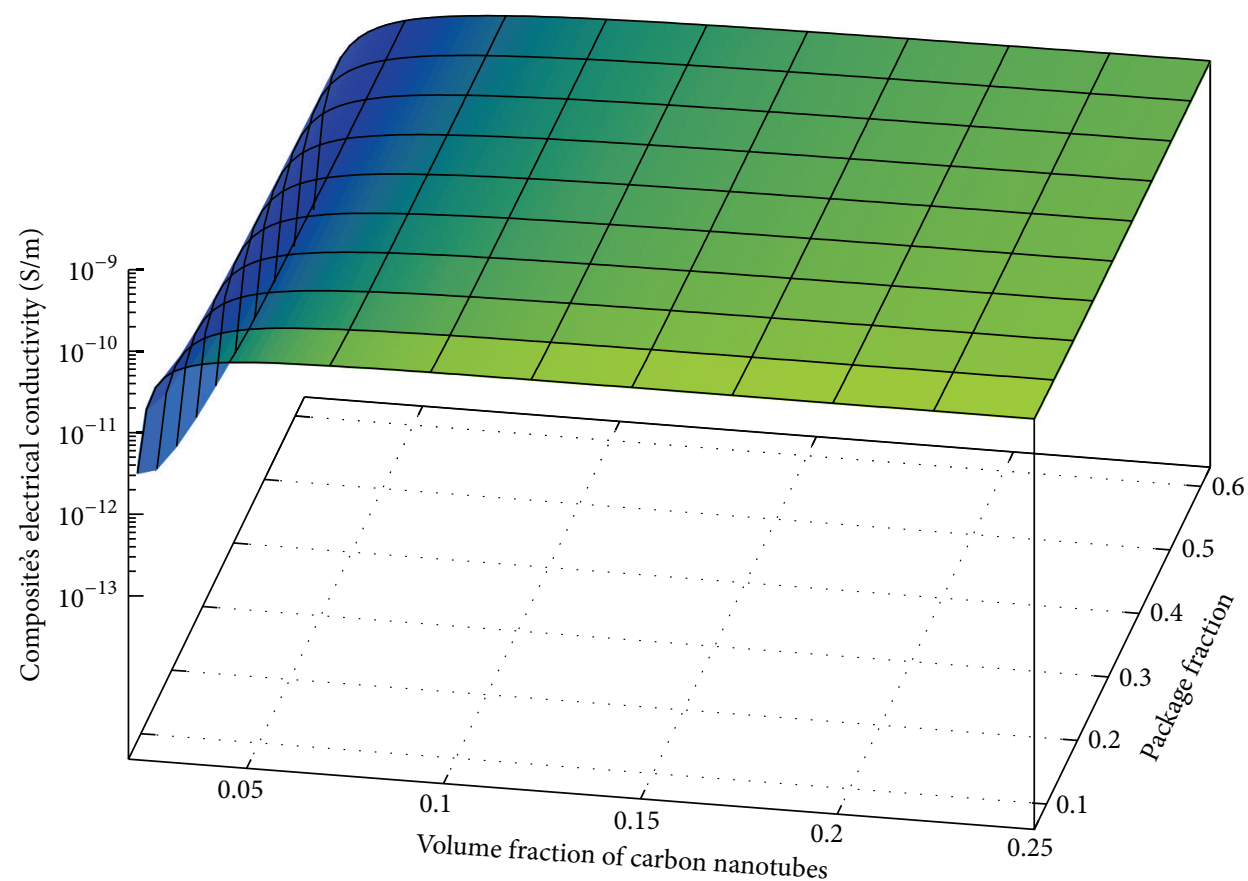

Figure 21: DC electrical conductivity of the nanocomposite at varying both the packaging fraction of the filler and the volume fraction of the filler (Mamunya's model).

parameters: midpoint of the percolation region's width, conductivity of the filler, and width of the percolation region. The volume fraction was restricted to the range of 0.02 to 0.25 . The midpoint of the percolation region's width was varied from 0.05 to 0.20 . The conductivity of the filler was varied from $1 E-15$ to $1 E-07 \mathrm{~S} / \mathrm{cm}$. The width of the percolation region was varied from $5 E-03$ to $45 E-03$.

\section{Results and Discussion}

In this section, the results and discussion of these models is realized. Each model is analyzed separately with the aim of identifying their advantages and drawbacks in the modeling of the nanocomposites. In addition, this analysis allow us determine which are the main parameters that must be considered when a design process related with this type of materials is used to fabricate electronic devices for VLSI circuits and systems.

4.1. Analysis of the Kirkpatrick's Model. In Figure 4, the variation of the electrical conductivity of composites with respect to the value of the critical exponent of the percolation is illustrated. Higher electrical conductivities are obtained when the value of the critical exponent is low. Therefore, this parameter must be controlled to manipulate the value of the electrical conductivity of the resulting composite.

The value of the conductivity of the filler determines the maximum electrical conductivity obtained for the composite as shown in Figure 5. Thus, this parameter can be reinforced as part of a strategy to increase the electrical conductivity of the nanocomposite to be designed.
At varying the percolation threshold of the filler in the composite, as shown in Figure 6, the electrical conductivity is not changed, but rather the initial value, for which an electrical conductivity is achieved, is displaced in accordance with the value of the percolation threshold.

In Figure 7, it is depicted that the behavior is obtained when both the critical component of the percolation and the volume fraction of the filler are modified. It was found that when critical exponent is low and volume fraction is high, high electrical conductivities are established. In the case of the conductivity of the filler and the volume fraction of the filler, as shown in Figure 8, the electrical conductivity of the composite is at maximum, if at least the first parameter is great. Finally, the percolation threshold at minimum and the volume fraction at maximum can increase the electrical conductivity of the nanocomposite, as depicted in Figure 9.

4.2. Analysis of the McLachlan's Model. The critical value of the percolation increases the electrical conductivity of the resulting nanocomposite with better intensity for greater values of the volume fraction, as shown in Figure 10. Thus, it is feasible considering that this parameter must be favored in the design of this class of materials.

The conductivity of the polymeric matrix can be considered as a value to be reinforced during the design of nanocomposites, since it increases the electrical conductivity of them as shown in Figure 11. Therefore, a better electrical conductivity of the matrix will lead to better conductive nanocomposites.

In Figure 12, at varying the percolation threshold of the filler, it was found that the initial value of the conductivity 


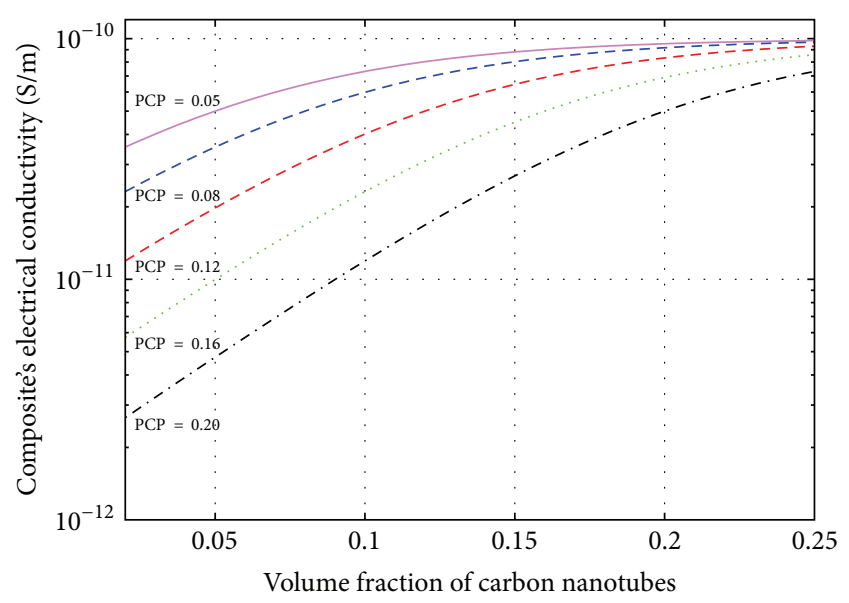

FIgURE 22: DC electrical conductivity of the nanocomposite when the midpoint of the percolation region's width is varied (Sigmoidal function model).

is restricted for values of the volume fraction greater to itself established in the simulation. Therefore, this parameter holds the increasing trend but the electrical conductivity is not favored.

Tridimensional changes in this model for three different parameters can be visualized in Figures 13, 14, and 15. In Figure 13, DC electrical conductivity is increased when critical exponent of the percolation and volume fraction of filler are bigger. The conductivity of the polymeric matrix intensifies the electrical conductivity of the composite if both it and the volume fraction are larger. When percolation threshold of the filler and volume fraction are varied, then the electrical conductivity of the nanocomposite is favored if both values are greater.

4.3. Analysis of the Mamunya's Model. In Figure 16, it is depicted that the change of DC electrical conductivity when the conductivity at the maximum packaging fraction is done. Therefore, it can be concluded that this parameter considerably increases electrical conductivity.

The conductivity at the percolation threshold uniquely modifies the initial value of the conductivity in the percolation threshold, as shown in Figure 17. Thus, an insignificant change in the electrical conductivity for all values of this parameter can be established.

The packaging fraction of the filler only changes the edge of the curve of the conductivity, but does not affect the maximum value of electrical conductivity of the nanocomposite, as shown in Figure 18.

In Figures 19, 20, and 21, the tridimensional changes of these behaviors are illustrated with the aim of emphasizing that the maximum electrical conductivity is achieved when the volume fraction and the conductivity at the maximum packaging fraction are great. Insignificant changes have been found when the conductivity at the percolation threshold of the filler and the packaging fraction are increased, since the maximum conductivity is achieved for almost any value of them.

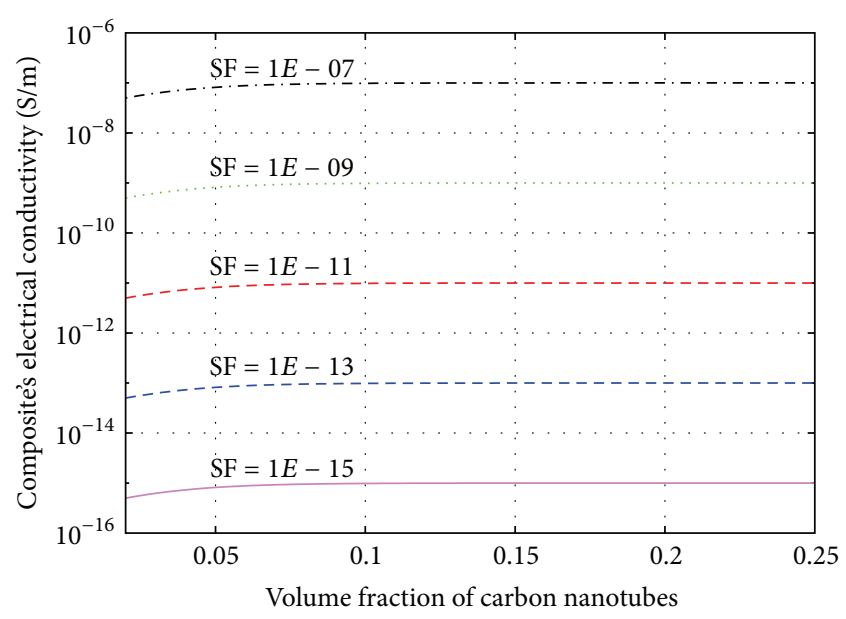

FIGURE 23: DC electrical conductivity of the nanocomposite at varying the conductivity of the filler (Sigmoidal function model).

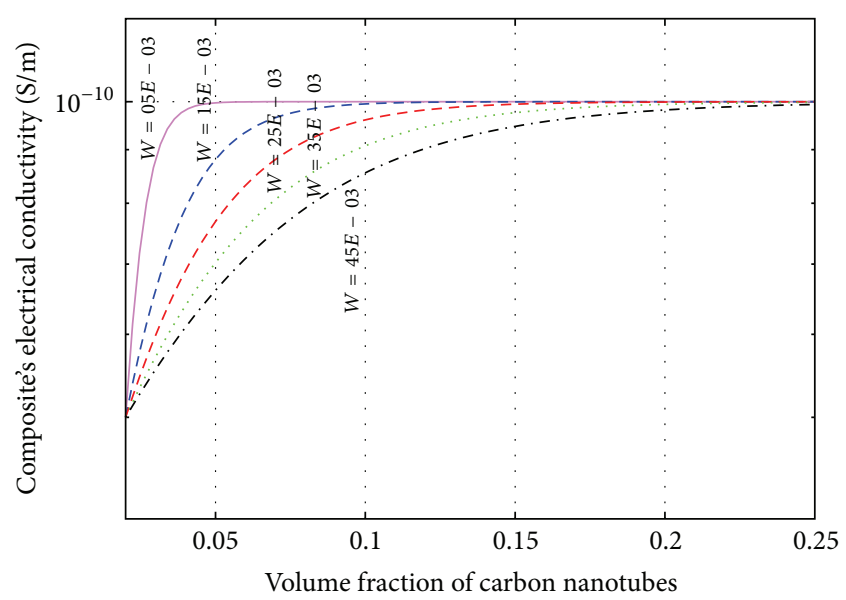

FIGURE 24: DC electrical conductivity of the nanocomposite when the width of the percolation region is varied (Sigmoidal function model).

4.4. Analysis of the Sigmoidal Function Model. In Figure 22, it can be observed that varying the midpoint of the percolation region's width modifies the initially electrical conductivity for small values of volume fraction, but the maximum value can be achieved for any value of the midpoint. Therefore, this value has an insignificant effect on the maximum value of the conductivity.

The conductivity of the filler has an important effect on the maximum value of the electrical conductivity of the nanocomposite, as shown in Figure 23. When the value of the conductivity of the filler is great, the conductivity also is increased. Thus, the filler's conductivity is a very important parameter in the design of these materials.

In the case of the width of the percolation region (Figure 24), it has a reduced effect on the range of values of the volume fraction of the filler since the slope of the curve is more abrupt or not, although the maximum value of the conductivity is not affected by this parameter. Therefore, this 


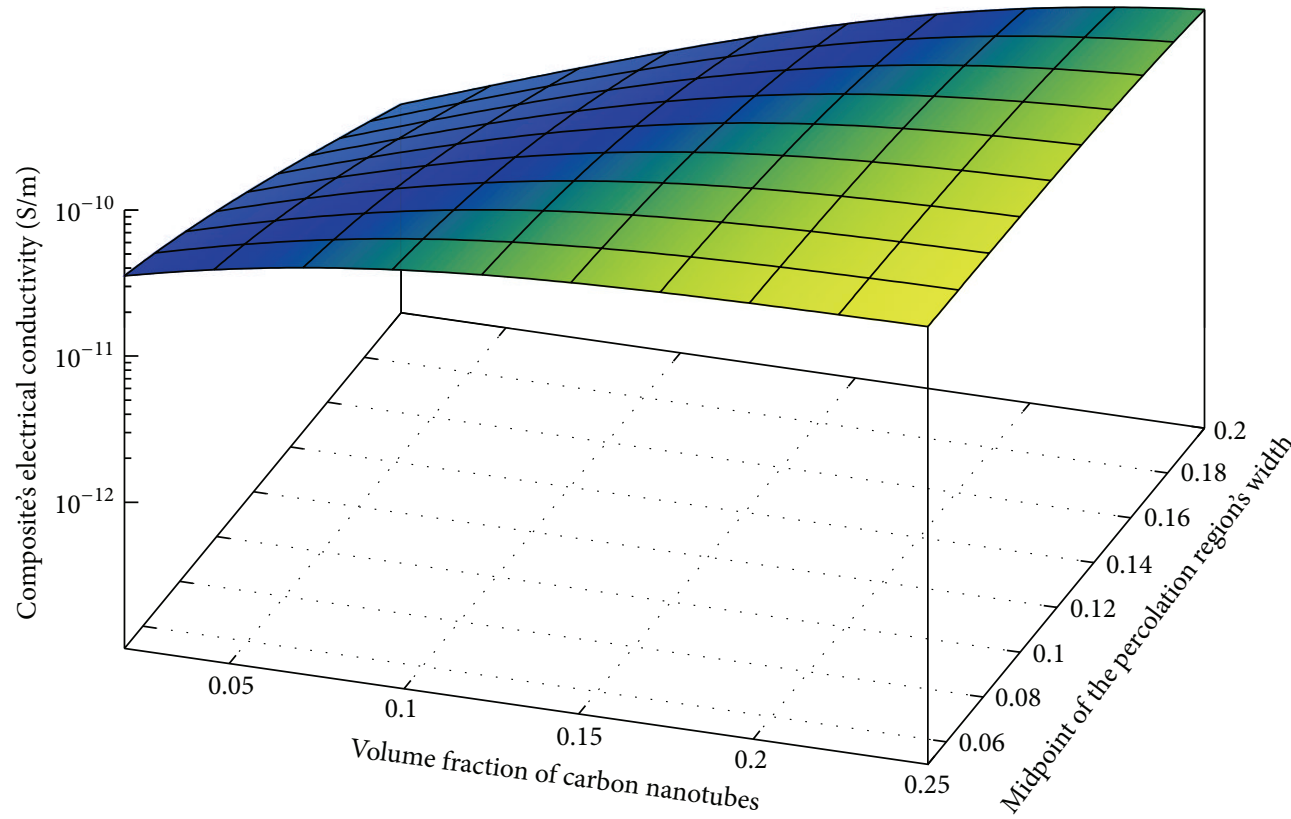

FIGURE 25: DC electrical conductivity of the nanocomposite at varying both the midpoint of the percolation region's width and the volume fraction of the filler (Sigmoidal function model).

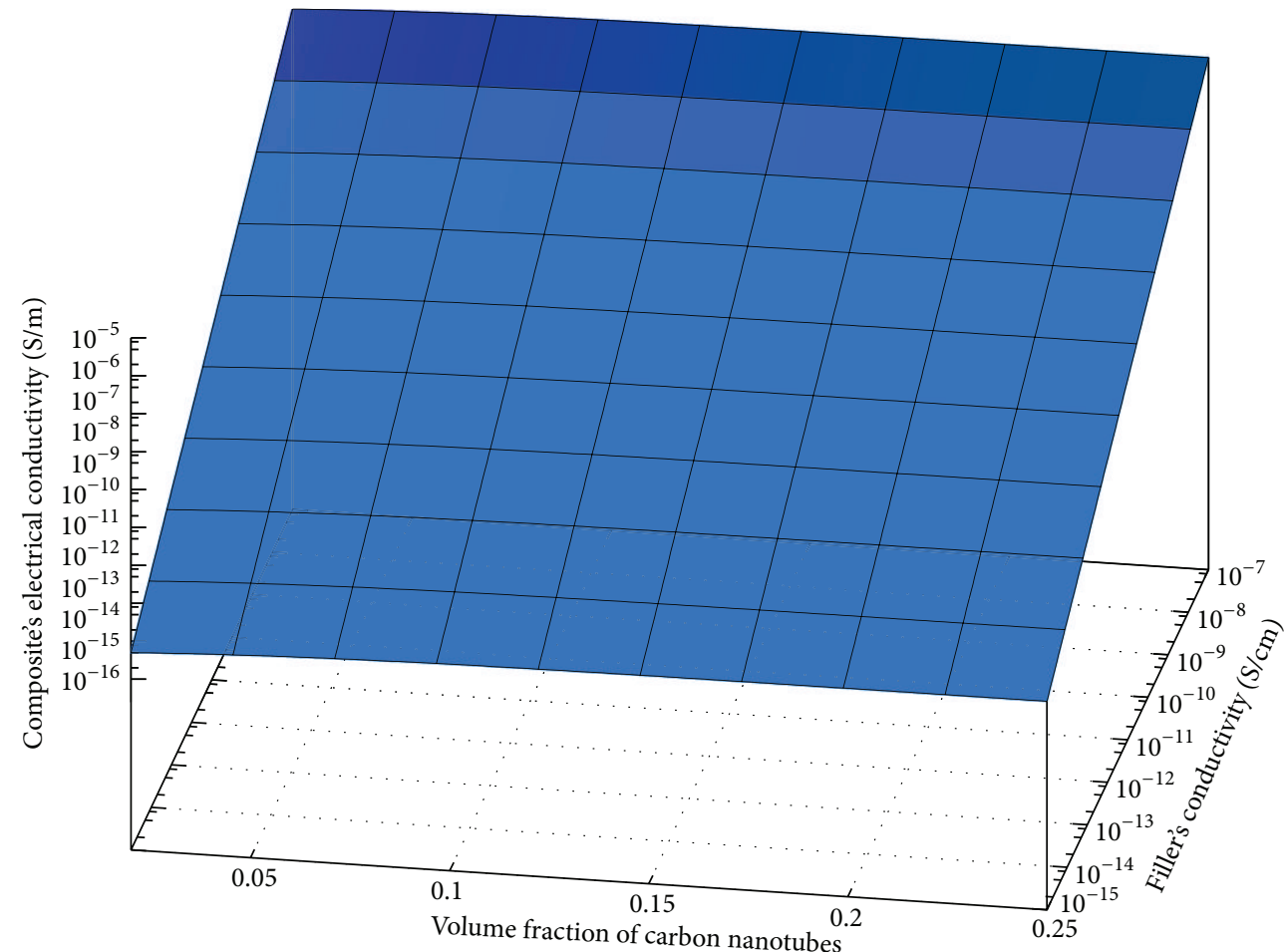

FIGURE 26: DC electrical conductivity of the nanocomposite when both the conductivity of the filler and the volume fraction of the filler are varied (Sigmoidal function model).

value has an insignificant effect on the maximum value of the conductivity of the nanocomposite.

In Figures 25, 26, and 27, a tridimensional representation of these changes allows the designer of these materials to take into account the regions where electrical conductivity will be maximum, middle, and minimum. In Figure 25, the maximum value of electrical conductivity is achieved when the volume fraction of the filler used was at maximum, and the midpoint of the percolations region's width is at maximum. The maximum value of conductivity can be 


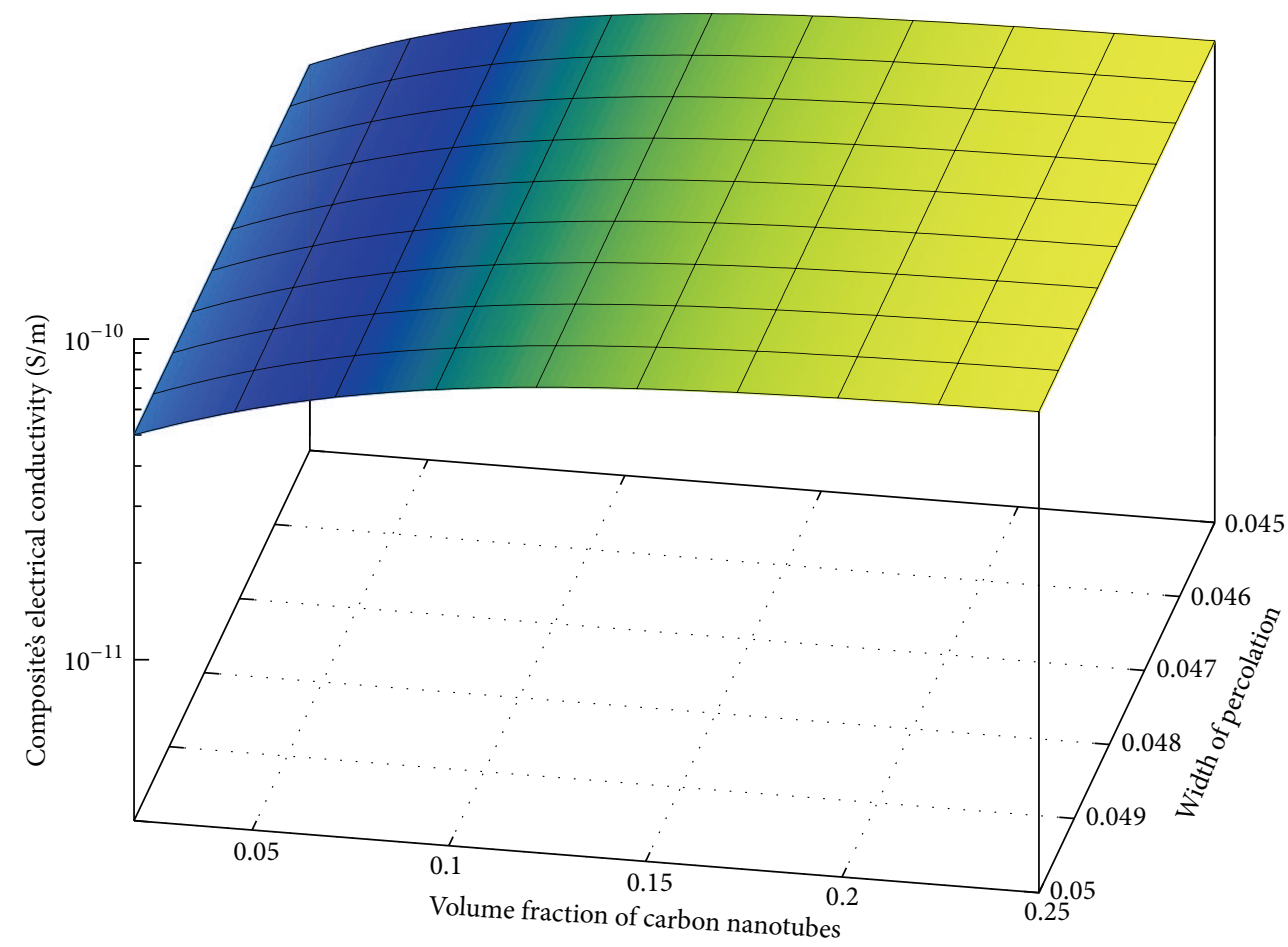

FIGURE 27: DC electrical conductivity of the nanocomposite at varying both the width of the percolation region and the volume fraction of the filler (Sigmoidal function model).

achieved when the conductivity of the filler is great, as shown in Figure 26. Finally, in Figure 27 it can be visualized that three different regions are found, but the maximum value is obtained when volume fraction is great.

4.5. Discussion of the Results. In Table 1 , it can be found that a recompilation of the effect of each one of the parameters associated with mathematical models is used to determine electrical conductivity of the composite materials based on carbon nanotubes and polymers. It is necessary to remember that volume fraction of carbon nanotubes in the composite is restricted in the range of 0.02 to 0.25 .

For Kirkpatrick's model, an increase in the electrical conductivity can be achieved. In the case of the McLachlan's model, a similar behavior can be found and specific values must be considered since two solutions can be obtained: (1) an increased behavior or (2) a decreased behavior. In addition, imaginary values in electrical conductivity can be achieved for determined ranges of parameters used in the mathematical model. These details have not been reported previously by different authors using this model. In particular, Mamunya's model has electrical conductivity restricted to the range where conductivity is at the maximum packaging fraction. Finally, Sigmoidal function model can be used for all values of concentration of the filler, and its behavior can be adjusted only when experimental values have been obtained since it belongs to the group of models based on nonlinear regression.

\section{Conclusions}

The DC electrical conductivity of nanocomposites based on polymers and carbon nanotubes has been studied with the aim of determining which are the main parameters of fabrication that must be considered for truly modifying the electrical behavior and how these change such behavior. This study was realized by computer simulation through four different models at varying three parameters in each one of them. Parameters such as volume fraction of the filler, percolation threshold of the filler, critical exponent of percolation, conductivity of the filler, conductivity of the matrix, conductivity at the maximum packaging fraction of the filler, conductivity at the percolation threshold, packaging fraction of the filler, midpoint of the percolation region's width, and width of the percolation region were analyzed. The main parameters involved in the increase of electrical conductivity of the nanocomposites are critical exponent of percolation, conductivity of the filler, conductivity of the matrix, conductivity at the maximum packaging fraction, conductivity at the percolation, and width of the percolation region. Unfortunately, a model that includes all these parameters does not exist until now. In McLachlan's model, specific values must be considered since two solutions can be possible: (1) an increased behavior or (2) a decreased behavior. In addition, imaginary values in electrical conductivity can be achieved for determined ranges of parameters used in the McLachlan's model. These details have not been reported previously by different authors using this model. 
The results presented here suggest the development of new electrical conductivity models for nanocomposites, where all these parameters are related with the aim of providing a better approach to the electrical behavior of these materials. A more sophisticated electrical model will allow to lead nanocomposites based on carbon nanotubes and polymers to the electronic industry for fabrication of VLSI circuits and systems.

\section{Acknowledgments}

This work was supported by the National Council of Science and Technology of Mexico (CONACYT) under project no.152524 under their support to basic research. The authors desire to thank the collaboration of Rodolfo Angulo Olais, M.S. (CINVESTAV-IPN Zacatenco, Mexico), Alfredo Aguilar Elguezabal, Ph.D. and José Martín Herrera Ramírez, Ph.D. (CIMAV-Chihuahua, México), Ana Arizmendi Morquecho (CIMAV-Monterrey, México), Bárbara Bermúdez Reyes, Ph.D. (Universidad Autónoma de Nuevo León, México), and Carlos A. De la Cruz-Blas, Ph.D. (Universidad Pública de Navarra, Spain).

\section{References}

[1] B. P. Grady, Carbon Nanotube-Polymer Composites: Manufacture, Properties, and Applications, John Wiley \& Sons, New York, NY, USA, 2011.

[2] N. Grossiord, J. Loos, L. Van Laake et al., "High-conductivity polymer nanocomposites obtained by tailoring the characteristics of carbon nanotube fillers," Advanced Functional Materials, vol. 18, no. 20, pp. 3226-3234, 2008.

[3] M. Sahimi, Applications of Percolation Theory, Taylor and Francis, Boca Raton, Fla, USA, 1994.

[4] S. Kirkpatrick, "Percolation and conduction," Reviews of Modern Physics, vol. 45, no. 4, pp. 574-588, 1973.

[5] R. Zallen, The Physics of Amorphous Solids, Wiley-Interscience, New York, NY, USA, 1998.

[6] D. Stauffer and A. Aharony, Introduction To Percolation Theory, Taylor \& Francis, London, UK, 2003.

[7] D. S. McLachlan, M. Blaszkiewicz, and R. E. Newnham, "Electrical resistivity of composites," Journal of the American Ceramic Society, vol. 73, no. 8, pp. 2187-2203, 1990.

[8] D. S. McLachlan, "Analytical functions for the DC and AC conductivity of conductor-insulator composites," Journal of Electroceramics, vol. 5, no. 2, pp. 93-110, 2000.

[9] D. S. McLachlan, C. Chiteme, C. Park et al., "AC and DC percolative conductivity of single wall carbon nanotube polymer composites," Journal of Polymer Science B, vol. 43, no. 22, pp. 3273-3287, 2005.

[10] R. L. Barton, J. M. Keith, and J. A. King, "Development and modeling of electrically conductive carbon filled liquid crystal polymer composites for fuel cell bipolar plate applications," Journal of New Materials for Electrochemical Systems, vol. 10, no. 4, pp. 225-229, 2007.

[11] D. S. McLachlan and G. Sauti, "The AC and DC conductivity of nanocomposites," Journal of Nanomaterials, Article ID 30389, 9 pages, 2007.
[12] E. P. Mamunya, V. V. Davidenko, and E. V. Lebedev, "Effect of polymer-filler interface interactions on percolation conductivity of thermoplastics filled with carbon black," Composite Interfaces, vol. 4, no. 4, pp. 169-176, 1997.

[13] Y. P. Mamunya, V. V. Davydenko, P. Pissis, and E. V. Lebedev, "Electrical and thermal conductivity of polymers filled with metal powders," European Polymer Journal, vol. 38, no. 9, pp. 1887-1897, 2002.

[14] J. M. Keith, J. A. King, and B. A. Johnson, "Electrical conductivity modeling of carbon filled polypropylene based resins for fuel cell bipolar plate applications," Journal of New Materials for Electrochemical Systems, vol. 11, no. 4, pp. 253-257, 2008.

[15] K. Kalaitzidou, H. Fukushima, and L. T. Drzal, "A route for polymer nanocomposites with engineered electrical conductivity and percolation threshold," Materials, vol. 3, no. 2, pp. 1089$1103,2010$.

[16] A. Merzouki and N. Haddaoui, "Electrical conductivity modeling of polypropylene composites filled with carbon black and acetylene black," ISRN Polymer Science, Article ID 493065, 7 pages, 2012.

[17] E. Tjørve, "Shapes and functions of species-area curves: a review of possible models," Journal of Biogeography, vol. 30, no. 6, pp. 827-835, 2003.

[18] F. A. Graybill and H. K. Iyer, Regression Analysis: Concepts and Applications, Duxbury Press, Belmont, Calif, USA, 1994.

[19] J. O. Rawlings, S. G. Pantula, and D. A. Dickey, Applied Regression Analysis: A Research Tool, Springer, New York, NY, USA, 1998.

[20] G. A. F. Seber and C. J. Wild, Nonlinear Regression, WileyInterscience, Hoboken, NJ, USA, 2003.

[21] S. Chatterjee and A. S. Hadi, Regression Analysis By Example, Wiley-Interscience, Hoboken, NJ, USA, 2006. 

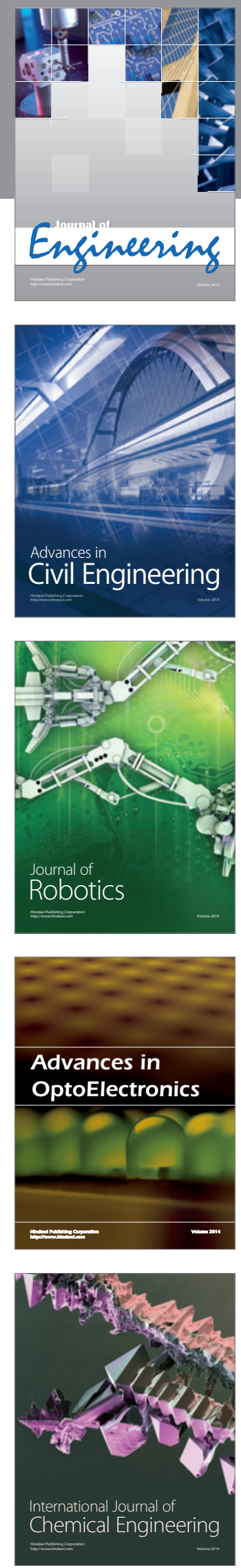

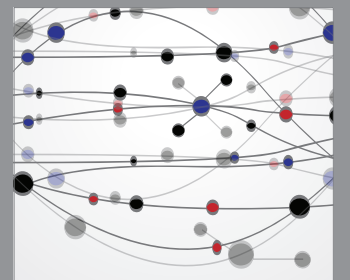

The Scientific World Journal
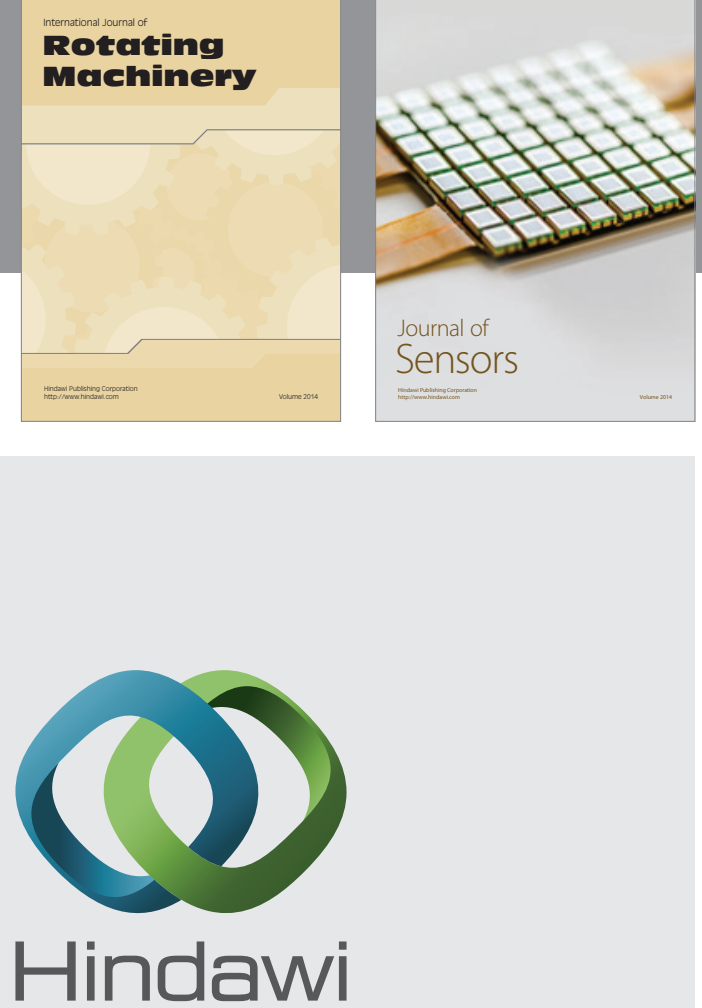

Submit your manuscripts at http://www.hindawi.com
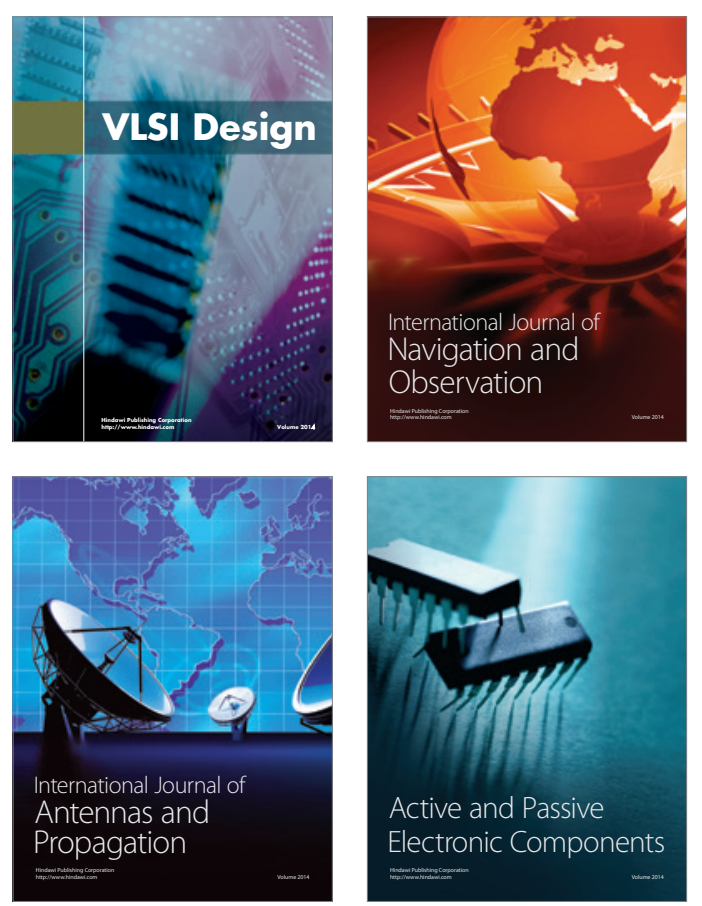
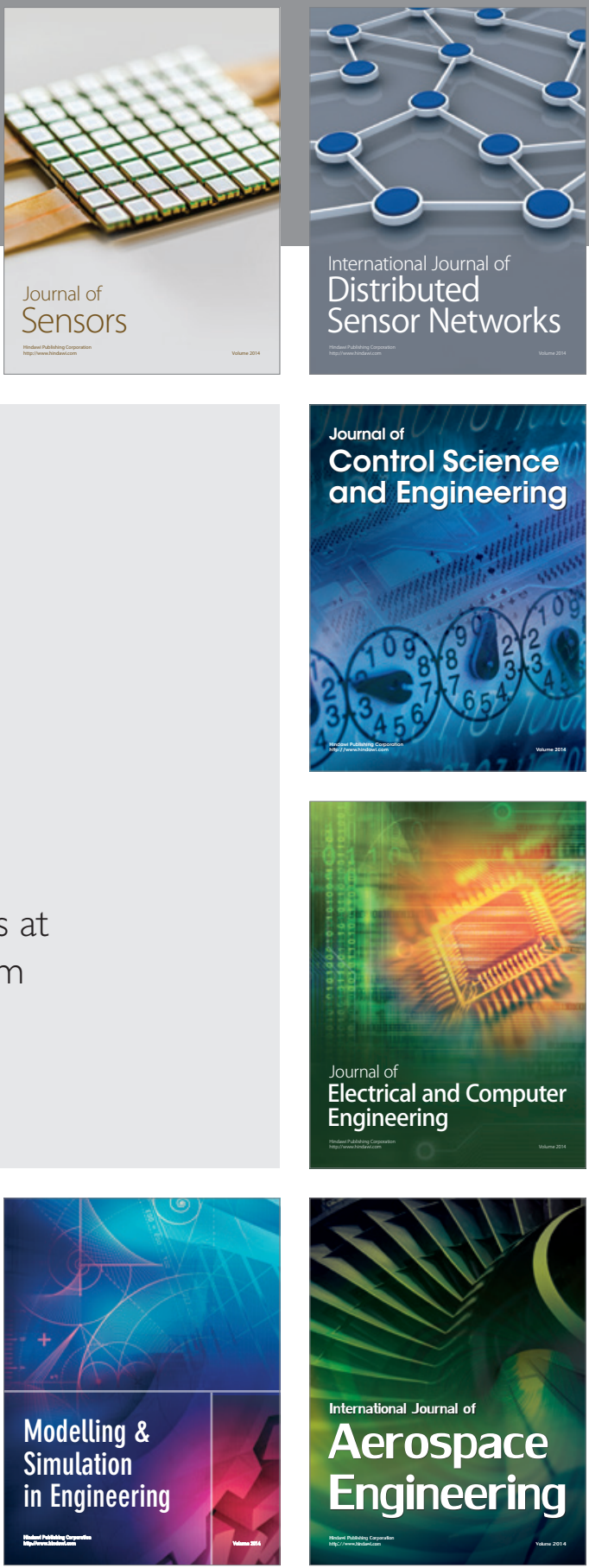

Journal of

Control Science

and Engineering
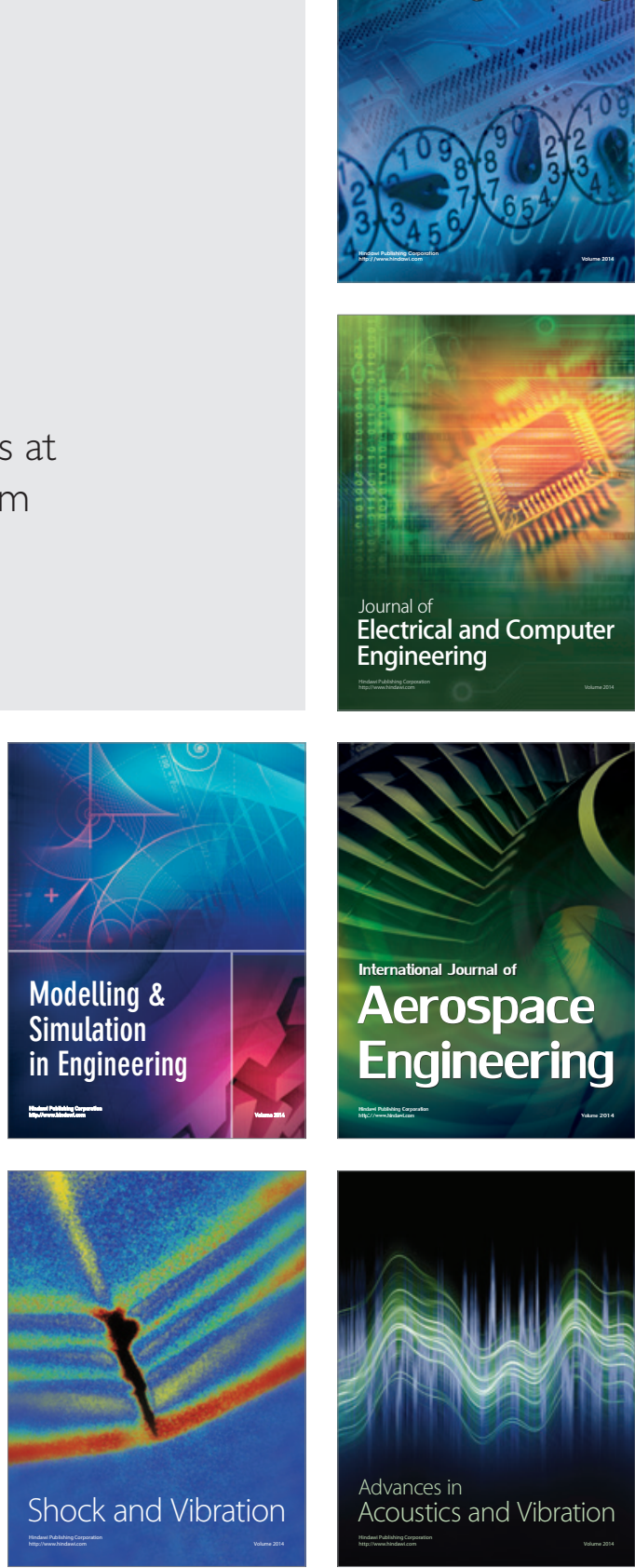